\title{
Experimental Investigation and Control of a Hot-Air Tunnel with Improved Performance and Energy Saving
}

\author{
Pavel Navrátil, Libor Pekař** Radek Matušo̊, Mengjie Song, Qingbin Gao, Shanti S. Kandala, \\ and Ondřej Kadlč́k
}

Cite This: ACS Omega 2021, 6, 16194-16215

Read Online

ACCESS | Lلlll Metrics \& More | 回 Article Recommendations | sl Supporting Information

ABSTRACT: The paper is focused on the identification, control design, and experimental verification of a two-input two-output hot-air laboratory apparatus representing a small-scale version of appliances widely used in the industry. A decentralized multivariable controller design is proposed, satisfying control-loop decoupling and measurable disturbance rejection. The proposed inverted or equivalent noninverted decoupling controllers serve for the rejection of cross-interactions in controlled loops, whereas open-loop antidisturbance members satisfy the absolute invariance to the disturbances. Explicit controller-structure design formulae are derived, and their equivalence to other decoupling schemes is proven. Three tuning rules are used to set primary controller parameters, which are further discretized. All the control responses are simulated in the Matlab/Simulink environment. In the experimental part,

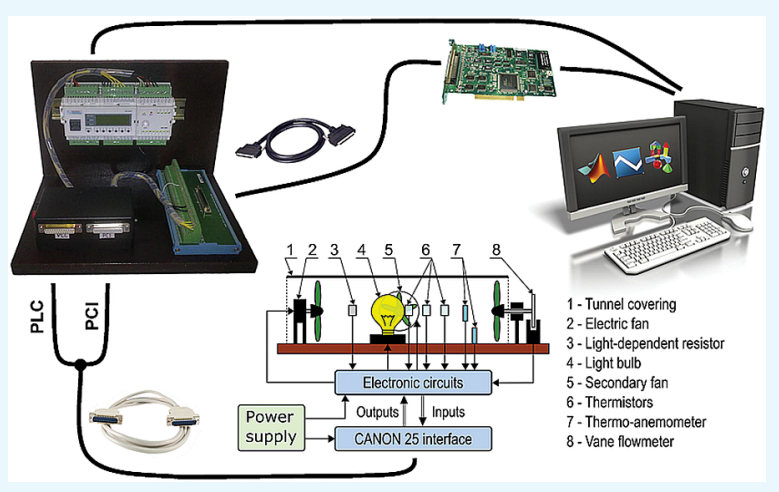
two data-acquisition, communication, and control interfaces are set up. Namely, a programmable logic controller and a computer equipped with the peripheral component interconnect card commonly used in industrial practice are implemented. A simple supervisory control and data acquisition human-machine interface via the Control Web environment is developed. The laboratory experiments prove better temperature control performance measured by integral criteria by $35.3 \%$, less energy consumption by up to $6 \%$, and control effort of mechanical actuator parts by up to $17.1 \%$ for our method compared to the coupled or disturbance-ignoring design in practice. It was also observed that the use of a programmable logic controller gives better performance measures for both temperature and air-flow control.

\section{INTRODUCTION}

Hot-air tunnels represent a family of direct-contact solid-gas heat exchangers that involve heat transfer between the solid heat source and the air stream where a separating wall is absent. ${ }^{1,2}$ These appliances of various constructions have been widely used in industry and commerce for decades. ${ }^{3}$ They are used for packaging (shrink wrap) of several products, ${ }^{4,5}$ when producing cosmetic containers with small dimensions, ${ }^{6}$ for thermal retraction of seals and cylindrical bottle made of glass, ${ }^{7}$ and for drying pigmented paints. ${ }^{8}$ The tunnels can also be found in rubber technology. ${ }^{9}$ For small cross-section profile manufacturing, hot-air-based vulcanization represents the most critical production system. ${ }^{10}$ They are used in the pharmaceutic industry for depyrogenation and sterilization. ${ }^{11}$

In the food industry, the tunnels are used for artificial convective hot-air drying on a commercial scale for numerous food products and have significant utilization in food production when dehydration. Morales-Delgado et al. ${ }^{12}$ and Doymaz ${ }^{13}$ observed several chemical and physical quantities of strawberries when drying in an experimental hot air tunnel dryer at different temperatures. The convective drying method using tunnels was studied on many fruits and vegetables such as ginger, chili, and button mushroom. ${ }^{14-17}$ The last step of the Aculon (i.e., a kind of hydrophobic chemical coating) treatment is the drying of the surfaces with dry air. ${ }^{18}$ Numerical models to predict the moisture and size of slices of gels containing gelatinized or native cornstarch and calcium alginate during convective drying performed in a tunnel were developed by Silva Júnior et al. ${ }^{19}$

Hot-air tunnel dryers are easy to construct and use, and they bring some benefits. Their advantage mainly consists of simple and robust construction and broad applicability. The acquisition and maintenance costs of tunnel dryers are low, allowing them to be used within small-scale drying operations in chemical engineering and processing. ${ }^{20}$ The drying of sweet potatoes helps prolong their storage life, and the dried form has a higher concentration of bioactive phytochemical

Received: April 28, 2021

Accepted: June 1, 2021

Published: June 11, 2021

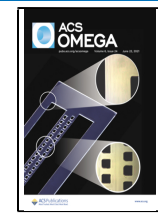


compounds than fresh sweet potatoes. ${ }^{21}$ The use of an elevated hot-air tunnel was found as a better option for drying coffee than a motorized stirrer or an electric pan. ${ }^{22}$ The solar dryer and tunnel dryer effect on the physicochemical properties of tomato powder was studied by Ghavidel and Davoodi, ${ }^{23}$ where the tunnel dryer was found to be a more effective method of drying as compared with solar drying.

Nevertheless, the use of conventional hot-air drying suffers from some drawbacks, and much improvement can be made for better operation of hot-air tunnels. Poor heat transfer yielding a long production line requirement is the principal disadvantage of hot-air tunnels. ${ }^{10}$ Yilmaz et al. ${ }^{24}$ recently reported that the most critical drawback of conventional dryings, such as hot-air drying, is an extended processing time at high temperatures, which results in a decrement of the dried product quality. When drying cassava, it was found that the energy efficiency of the tunnel dryer was almost twice less than that of the pneumatic dryer, ${ }^{25}$ where most of the heat losses were through unsaturated exhaust air. The amount of polyphenolics, 2,2-diphenyl-1-picrylhydrazyl, and proteins was found to be lower when tunnel drying compared to freeze or vacuum drying yet higher than that for the solar dryer when assessing the effect of various dehydration techniques on the physicochemical and nutritional properties of the guava powder. ${ }^{26}$

The above-introduced deficiencies, especially the performance and energy consumption of the hot-air-tunnel (drying) process, make it reasonable to investigate further improvements in control temperature and heat flux inside the tunnel. These processes ought to be considered and modeled as a multivariable system since the input hot-air flow driven by a fan affects not only the output flow but also the output temperature. However, the laboratory appliance used in this research has been considered solely as the single-input singleoutput (SISO) process when performing control tasks, regarding either temperature control ${ }^{27}$ or the air-flow speed control. $^{28}$ Pivoňka and Nepevný ${ }^{29}$ applied a multi-input multioutput (MIMO) neural-network model-based predictive controller to a different physical model yet with very similar functionality to the considered one. A programmable logic controller (PLC) served only as a communication interface, and the effect of disturbances was not analyzed therein. Model-based predictive and fuzzy control principles were also used when controlling the ventilation system of a road tunnel. $^{30}$ In fact, the required number of jet fans was considered as the only manipulated input therein. Longitudinally ventilated road tunnels attract researchers mainly due to the investigation, modeling, and control of fire-induced smoke and heat movement where the temperature inside, ventilation velocity, maximum heat flux, oxygen concentration, etc. are measured. ${ }^{31-33}$ However, ad hoc recommendation and manipulation patterns rather than rigorous control algorithms for ventilation have been provided. Temperature control serves to achieve the desired shrinkage and relief markings ${ }^{34}$ when producing commodities covered by shrink polymeric materials inside hot-air tunnels; nevertheless, particular control laws remain unknown. Some researchers investigated the microclimate of greenhouses, as a subclass of hot-air tunnels, for control purposes. ${ }^{35}$ As climatic conditions (temperature and humidity) inside greenhouses are very complex and nonlinear, their control is particularly challenging. ${ }^{36}$ Jung et al. ${ }^{37}$ proposed an output feedback neuralnetwork ventilation control method for greenhouses. The authors claimed that control algorithms based on the proportional-integral-derivative (PID) controllers are difficult to apply effectively due to the necessity of suitable parameters' tuning and the delay effect on actuators. Therefore, control logic utilizing the user's experience or energy-conservation laws is mainly used. ${ }^{38-40}$

Regarding general MIMO control systems, a multitude of ideas and research results have been investigated and published. Compared to SISO plants, they are more challenging to control due to interdependence and crossinteractions between inputs and outputs. There are two fundamental ways of realizing a control strategy for MIMO systems effectively. First, one can use a centralized controller with antidisturbance decouplers; second, decentralized controllers can be designed, with or without terms decoupling unwanted cross-interaction. ${ }^{41}$ Decentralized control ${ }^{42-45}$ uses independent SISO controllers that act on particular manipulated inputs. Contrariwise, centralized control represents a framework strategy exploiting a MIMO feedback controller, ${ }^{46-48}$ i.e., it needs to consider all input-output controller pairs when designing a control law. A simpler tuning, more straightforward implementation, better robustness, or more efficient maintenance pose the advantages of decentralized control compared to a centralized one. ${ }^{42}$ However, although a decentralized control configuration is set, the controlled plant still includes coupled pairs, which yields cross-interactions in the control loop. Decoupling elements (or decouplers) then should ensure that every single control loop has no interaction with another one. ${ }^{41,49}$ The ideal decouplers attempt to reach a diagonal open-loop serial link between the decoupler and the control system, i.e., the open loop is described by independent SISO transfer functions that are identical to selected entries of the plant transfer function matrix. ${ }^{50-52}$ However, many design frameworks resulted in nonideal or partially coupled decoupling schemes. This fact is given either by the nature of the design (e.g., for static ${ }^{53}$ or simplified ${ }^{45,50,54}$ decouplers) or it yields from the particular design for systems with complicated behavior (e.g., for nonminimum phase plants. ${ }^{46,48,55}$ In practice, numerous combinations of centralized/decentralized control principles (including fuzzy, bioinspired, artificialneural-network-based, etc.) with decoupling schemes have been recently proposed, for several types of systems. $45,47,48,56-58$

It is necessary for decentralized control and decoupling to select appropriate input-output pairs to be controlled while the remaining ones are neglected. This operation called the "pairing selection" aims to minimize the number of loop crossinteractions. It is worth noting that even if the controllers are tuned well, inappropriate pairing selection may lead to poor closed-loop performance or its instability. ${ }^{59}$ Various interaction measures and pairing criteria have been proposed, e.g., the relative gain array (RGA), ${ }^{59,60}$ dynamic RGA, ${ }^{61}$ absolute $\mathrm{RGA}^{62}$ normalized RGA (NRGA), ${ }^{63}$ fuzzy-logic-based optimal pairing, ${ }^{64}$ etc. The reader is referred to the work by Liu et al. ${ }^{41}$ for an overview of decoupling schemes and pairing selection.

We have proposed ${ }^{65}$ an inverted decentralized MIMO control design with a combination of the RGA, the NRGA, and the Niederlinski index ${ }^{66}$ to get optimal control pairs. It was proved that the inverted decoupler coincides with that designed by Garrido et al. ${ }^{46}$ The method was verified and compared with some other approaches ${ }^{45,46,48,64}$ using a model 
of a quadruple tank, providing explicit formulae for designing decouplers, better performance compared to nonoptimal pairings, and selected control techniques. It was shown in practice $^{41,55}$ that an inverted decoupling scheme has some advantages. For instance, wind-up compensators are easy to construct, the acting of decoupled loops can be kept away from the others, or decoupling is not affected by switching from/to the manual mode. On the contrary, when using it for delayed or nonminimum phase systems, it is necessary to compensate for nonfeasible or unstable factors in transfer function matrix entries.

In this paper, we adopt the idea of the above-referred recently developed decentralized decoupling method ${ }^{65}$ and apply it to control a laboratory hot-air tunnel that represents a small-scale model of existing industrial appliances. A two-input two-output (TITO) model was chosen where input voltages to the inlet heat source and the main electric fan are taken as manipulated inputs, whereas the control outputs are made up by the measured voltage on a thermometer near the heat source and by the voltage on the outlet vane flowmeter. Model parameter values are identified via the standard System Identification Toolbox in Matlab and by the least mean square (LMS) method for the ARX (autoregressive with exogenous) model for the comparison. The inverted decoupler in the form of the auxiliary controller transfer function matrix is used, for which the equivalent noninverted one is introduced. Once the loop interactions are decoupled, primary feedback controller parameters are set using the balanced tuning, ${ }^{67}$ the desiredmodel, $^{68}$ and a polynomial ${ }^{69,70}$ methods. In addition, measurable load disturbances-in the form of the air flux caused by a lateral fan-are fully compensated by feedforward correction matrices to get the so-called absolute invariance. The control system performance is benchmarked by simulations in the Matlab/Simulink environment via some integral criteria and compared to the control loop without decoupling and disturbance-rejection terms.

The experimental part of this research is substantial since it provides a real-life verification of the proposed method and a simple comparative study. The control loop is realized by using two different control devices. First, the standard personal computer (PC) equipped with the peripheral component interconnect (PCI) card (which is commonly used in industry for communication, data acquisition, and control tasks) is employed. Discretized controller laws are realized in Matlab/Simulink equipped with Real Time Windows Target library. Second, the eventual discrete-time controllers are implemented on a programmable logic controller (PLC), for which a simple SCADA/HMI in the Control Web environment is developed. Real-time verification and comparative experiments are performed to expose and demonstrate the advantages and drawbacks of the proposed method.

This work motivation is fourfold. First, there is an endeavor to enhance overall tunnel control performance, energy efficiency, and control effort compared to coupled feedback MIMO approaches. Second, as PID controllers prevail in industrial practice so far, and hence, engineers are sufficiently experienced to work with them, we want to verify the usability and performance of this class of controllers for several tuning rules. Third, the PLC and the PCI cards inside industrial computers are commonly used as control devices; therefore, it is desirable to implement the designed control system via those appliances. Last but not least, several comparisons are made numerically, the results of which can be applied in engineering practice. Namely, the designed decoupling and disturbance-rejection elements are compared to the basic scheme without these terms. Besides, we benchmark different controller parameter settings, continuous-time control law formulations versus discrete-time, and the use of the PLC compared to the application of the PCI card.

The contribution of this study can be summarized as follows:

1 A TITO (not SISO) controller is designed for the hotair tunnel model since the air-flow rate also affects the output temperature value. Moreover, the combination of both the controlled quantities determines the air moisture, which can be further used for other technological purposes.

2 A novel inverted decoupler and its noninverted equivalent form are used to assure the ideal absolute invariance. Explicit decoupler setting rules are given. Although the continuous-time disturbance-rejection law is not feasible, its digital implementation can be physically applied.

3 The whole design is experimentally verified via two different control devices commonly used in practice. In addition, a simple human-machine interface is developed to support the task.

4 The proposed method gives better performance than the simple control-feedback system, and improved energy- and actuator-saving behavior is demonstrated.

It is worth noting that our intention is not to determine suitable or optimal temperature or air-flow setpoints, which should be determined by process and chemical engineers for a particular process in the industry.

The rest of the paper is organized as follows: Section 2 introduces the designed multivariable control loop. Explicit setting rules for the proposed inverted decoupler and the disturbance-rejection controller are derived. The equivalences between the decoupler, its noninverted version, and the standard inverted decoupler are proven. Selected wellestablished identification techniques and tools and SISO PI(D) controller tuning rules are summarized therein. Feedback robust stability in terms of unstructured multiplicative input uncertainties is also concisely introduced in this section. In Section 3, descriptions of the hot-air tunnel, the used PCI card, and the applied PLC are given to provide the reader with the experimental setup. Experimental identification of the hot-air tunnel model parameters, followed by control synthesis, controller parameter tuning, and the used discretization technique are presented in Section 4. In this section, the obtained simulated and measured control responses, the developed SCADA/HMI, and the evaluation using selected integral criteria are provided. The results are compared to those obtained by the simple feedback loop without a decoupler and a disturbance-rejection subsystem. Then, the paper is concluded in Section 5. Some figures are provided in the Supporting Information for better readability.

\section{THEORETICAL METHODS AND RESULTS}

2.1. Proposed MIMO Control System Description. Consider a square linear time-invariant MIMO controlled plant with the manipulated input and output vectors $\mathbf{u}(t) \in \mathbb{R}^{n}$ and $\mathbf{y}(t) \in \mathbb{R}^{n}$, respectively. Let the plant be affected by (measurable) load disturbances $\mathbf{d}(t) \in \mathbb{R}^{m}, m \leq n$ 
. Denote by $\mathbf{u}(s), \mathbf{y}(s)$, and $\mathbf{d}(s)$ their Laplace transforms that give rise to the following relation:

$$
\mathbf{y}(s)=\mathbf{G}_{\mathrm{S}}(s) \mathbf{u}(s)+\mathbf{G}_{\mathrm{SD}}(s) \mathbf{d}(s)
$$

where $\mathbf{G}_{\mathrm{S}}(s)=\left[S_{i j}(s)\right]_{n \times n}$ and $\mathbf{G}_{\mathrm{SD}}(s)=\left[\mathrm{SD}_{i j}(s)\right]_{n \times m}$ are the corresponding transfer function matrices.

The proposed feedback control system is depicted in Figure 1 , where $\mathbf{G}_{\mathrm{C}}(s)=\left[C_{i j}(s)\right]_{n \times n}$ stands for the primary controller,

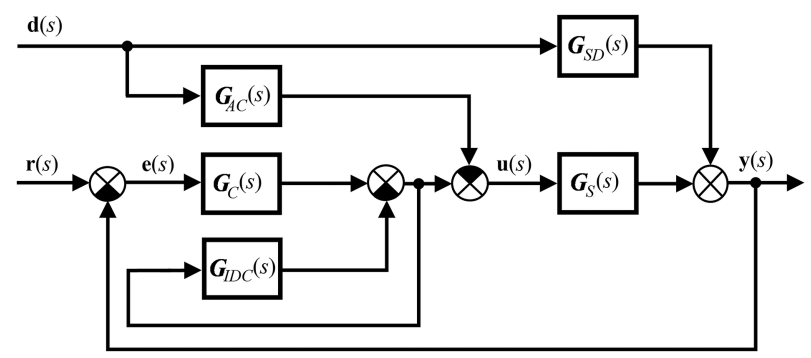

Figure 1. Proposed MIMO control system with the designed inverted decoupler.

$\mathbf{G}_{\mathrm{AC}}(s)=\left[\mathrm{AC}_{i j}(s)\right]_{n \times m}$ is the antidisturbance controller responsible for measurable disturbance rejection, and $\mathbf{G}_{\mathrm{IDC}}(s)=\left[\operatorname{IDC}_{i j}(s)\right]_{n \times n}$ represents the inverted decoupler that ensures the rejection of all undesirable input-output cross-interaction. The external signal $\mathbf{r}(s) \in \mathbb{R}^{n}$ means the Laplace form of the vector of references and $\mathbf{e}(s)=\mathbf{r}(s)-$ $\mathbf{y}(s)$ is that of the control errors.

The feedback transfer function matrices and their relations read

$$
\begin{aligned}
\mathbf{y}(s) & =\mathbf{G}_{\mathrm{RY}}(s) \mathbf{r}(s) \\
& =\left[\mathbf{I}+\mathbf{G}_{\mathrm{S}}(s) \mathbf{G}_{\mathrm{D}}(s) \mathbf{G}_{\mathrm{C}}(s)\right]^{-1} \mathbf{G}_{\mathrm{S}}(s) \mathbf{G}_{\mathrm{D}}(s) \mathbf{G}_{\mathrm{C}}(s) \mathbf{r}(s)
\end{aligned}
$$

for $\mathbf{d}(s)=0$ and

$$
\begin{aligned}
\mathbf{y}(s)= & \mathbf{G}_{\mathrm{DY}}(s) \mathbf{d}(s) \\
= & {\left[\mathbf{I}+\mathbf{G}_{\mathrm{S}}(s) \mathbf{G}_{\mathrm{D}}(s) \mathbf{G}_{\mathrm{C}}(s)\right]^{-1}\left[\mathbf{G}_{\mathrm{SD}}(s)-\mathbf{G}_{\mathrm{S}}(s) \mathbf{G}_{\mathrm{AC}}(s)\right] } \\
& \mathbf{d}(s)
\end{aligned}
$$

for $\mathbf{r}(s)=0$, where $\mathbf{I} \in \mathbb{R}^{n \times n}$ expresses the identity matrix, and $\mathbf{G}_{\mathrm{D}}(s)$ is the overall transfer function matrix of the decoupling subsystem, the form of which related to Figure 1 reads

$$
\mathbf{G}_{\mathrm{D}}(s)=\left[\mathbf{I}+\mathbf{G}_{\mathrm{IDC}}(s)\right]^{-1}
$$

The introduction of the inverted decoupler is motivated by the transition from the open-loop to a feedback control system. Such a scheme has some advantages, ${ }^{41,55,65}$ see Section 1. The proposed inverted decoupling scheme can be matched with the simple noninverted decoupler and the standard inverted decoupler. ${ }^{46}$ Mutual equivalent relations between these three schemes are shown in the following subsection.

2.2. Decoupling Scheme Equivalence. Control feedback loops with the (simple) noninverted and the wellestablished inverted decoupler proposed by Garrido et al. ${ }^{46}$ are displayed in Figure 2a,b, respectively, governed by

$$
\mathbf{G}_{\mathrm{D}}(s)=\mathbf{G}_{\mathrm{DC}}(s)
$$

$$
\mathbf{G}_{\mathrm{D}}(s)=\mathbf{G}_{\mathrm{Dd}}(s)\left[\mathbf{I}-\mathbf{G}_{\mathrm{Do}}(s) \mathbf{G}_{\mathrm{Dd}}(s)\right]^{-1}
$$

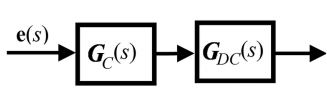

(a)

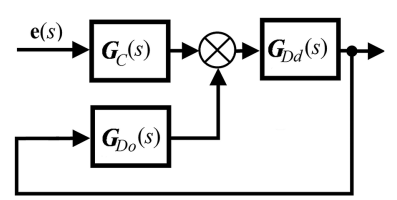

(b)
Figure 2. Simple noninverted decoupler (a) and standard inverted decoupler (b).

Proposition 1. Consider decoupler transfer functions (4)(6). Then

$$
\begin{aligned}
& \mathbf{G}_{\mathrm{IDC}}(s)=\mathbf{G}_{\mathrm{DC}}^{-1}(s)-\mathbf{I} \\
& \mathbf{G}_{\mathrm{IDC}}(s)=\mathbf{G}_{\mathrm{Dd}}^{-1}(s)-\mathbf{G}_{\mathrm{Do}}(s)-\mathbf{I}
\end{aligned}
$$

Proof. By comparison of (4) and (5)

$$
\left[\mathbf{I}+\mathbf{G}_{\mathrm{IDC}}(s)\right]^{-1}=\mathbf{G}_{\mathrm{DC}} \Leftrightarrow \mathbf{I}+\mathbf{G}_{\mathrm{IDC}}(s)=\mathbf{G}_{\mathrm{DC}}^{-1}
$$

which yields (7a) directly. Analogously, the following chain of identities can be obtained from the matching (4) and (6):

$$
\begin{gathered}
{\left[\mathbf{I}+\mathbf{G}_{\mathrm{IDC}}(s)\right]^{-1}=\mathbf{G}_{\mathrm{Dd}}(s)\left[\mathbf{I}-\mathbf{G}_{\mathrm{Do}}(s) \mathbf{G}_{\mathrm{Dd}}(s)\right]^{-1}} \\
\begin{aligned}
\mathbf{I}+\mathbf{G}_{\mathrm{IDC}}(s) & =\left[\mathbf{I}-\mathbf{G}_{\mathrm{Do}}(s) \mathbf{G}_{\mathrm{Dd}}(s)\right] \mathbf{G}_{\mathrm{Dd}}^{-1}(s) \\
& =\mathbf{G}_{\mathrm{Dd}}^{-1}(s)-\mathbf{G}_{\mathrm{Do}}(s)
\end{aligned} \\
\mathbf{G}_{\mathrm{IDC}}(s)=\mathbf{G}_{\mathrm{Dd}}^{-1}(s)-\mathbf{G}_{\mathrm{Do}}(s)-\mathbf{I}
\end{gathered}
$$

which agrees with $(7 \mathrm{~b})$.

2.3. Decoupling Subsystem Design. The goal of decoupling can be expressed by the condition

$$
\mathbf{G}_{\mathrm{S}}(s) \mathbf{G}_{\mathrm{D}}(s)=\overline{\mathbf{G}}_{\mathrm{S}}(s)
$$

where $\overline{\mathbf{G}}_{\mathrm{S}}(s)$ represents the desired open-loop controlled transfer function matrix determining the set of SISO control pairs. That is, an element $\bar{S}_{i j}(s)$ of $\overline{\mathbf{G}}_{\mathrm{S}}(s)$ is nonzero if and only if $y_{i}-u_{j}$ is the dominant control pair of the plant. Let us denote by $\left(i_{k}, j\right)$ the position of the element of $\mathbf{G}_{\mathrm{S}}(s)$ in the $j$ th column where $i_{k}$ means the row index of the dominant element in the $k$ th column. In other words, if $k=j$, then the element $S_{i_{j}}(s)$ is dominant.

The ideal decoupling means

$$
\overline{\mathbf{G}}_{\mathrm{S}}(s)=\mathbf{G}_{\mathrm{S}}(s) \odot \mathbf{M}
$$

where an element $M_{i j}=1$ of $\mathbf{M} \in \mathbb{R}^{n \times n}$ if $y_{i}-u_{j}$ is the dominant control pair, else $M_{i j}=0$, and $\odot$ expresses the Schur (Hadamard) product. Otherwise, the decoupling is nonideal (simplified).

Consider the noninverted decoupler (5) first.

Proposition 2. Decoupler $\mathbf{G}_{\mathrm{DC}}(s)=\left[\mathrm{DC}_{i j}(s)\right]_{n \times n}$ satisfies condition (8) if

$$
\mathrm{DC}_{i j}(s)=\frac{\mathrm{Sc}_{i_{j} i}}{\mathrm{Sc}_{i_{j} n}} \mathrm{DC}_{n j}(s), \quad i=1,2, \ldots, n-1 ; j=1,2, \ldots, n
$$

where $\mathrm{Sc}_{i j}$ is the cofactor of $S_{i j}$. Moreover, if

$$
\mathrm{DC}_{n j}(s)=\frac{1}{\operatorname{det} \mathbf{G}_{\mathrm{S}}(s)} \mathrm{Sc}_{i_{j} n} \bar{S}_{i_{j} j}, \quad j=1,2, \ldots, n
$$


where $\bar{S}_{i, j}$ is taken as in (9), then the ideal decoupling is obtained. Otherwise, a simplified decoupler is reached.

Proof. The element-by-element formulation of (8) reads

$$
\begin{aligned}
& \mathbf{G}_{\mathrm{DC}}(s)=\mathbf{G}_{\mathrm{S}}^{-1}(s) \overline{\mathbf{G}}_{\mathrm{S}}(s)\left[\begin{array}{ccccc}
\mathrm{DC}_{11}(s) & \mathrm{DC}_{12}(s) & \cdots & \cdots & \mathrm{DC}_{1 n}(s) \\
\mathrm{DC}_{21}(s) & \mathrm{DC}_{22}(s) & \cdots & \cdots & \mathrm{DC}_{2 n}(s) \\
\vdots & \vdots & \ddots & \ddots & \vdots \\
\vdots & \vdots & \ddots & \ddots & \vdots \\
\mathrm{DC}_{n 1}(s) & \mathrm{DC}_{n 2}(s) & \cdots & \cdots & \mathrm{DC}_{n n}(s)
\end{array}\right] \\
& =\frac{1}{\operatorname{det} \mathbf{G}_{\mathrm{S}}(s)}\left[\begin{array}{ccccc}
\mathrm{Sc}_{11}(s) & \mathrm{Sc}_{21}(s) & \cdots & \cdots & \mathrm{Sc}_{n 1}(s) \\
\mathrm{Sc}_{12}(s) & \mathrm{Sc}_{22}(s) & \cdots & \cdots & \mathrm{Sc}_{n 2}(s) \\
\vdots & \vdots & \ddots & \ddots & \vdots \\
\vdots & \vdots & \ddots & \ddots & \vdots \\
\operatorname{Sc}_{1 n}(s) & \mathrm{Sc}_{2 n}(s) & \cdots & \cdots & \mathrm{Sc}_{n n}(s)
\end{array}\right] \\
& {\left[\begin{array}{ccccc}
0 & 0 & \cdots & \cdots & 0 \\
\vdots & \vdots & \cdots & \cdots & \vdots \\
\bar{S}_{i_{1} 1}(s) & \bar{S}_{i_{2} 2}(s) & \ddots & \ddots & \bar{S}_{i_{n} n}(s) \\
\vdots & \vdots & \ddots & \ddots & \vdots \\
0 & 0 & \cdots & \cdots & 0
\end{array}\right]} \\
& =\frac{1}{\operatorname{det} \mathbf{G}_{\mathrm{S}}(s)}\left[\begin{array}{ccccc}
\mathrm{Sc}_{i_{1} 1}(s) \bar{S}_{i_{1} 1}(s) & \mathrm{Sc}_{i_{2} 1}(s) \bar{S}_{i_{2} 2}(s) & \cdots & \cdots & \mathrm{Sc}_{i_{i_{n} 1}}(s) \bar{S}_{i_{i^{n}}}(s) \\
\mathrm{Sc}_{i_{1} 2}(s) \bar{S}_{i_{1} 1}(s) & \mathrm{Sc}_{i_{2} 2}(s) \bar{S}_{i_{2} 2}(s) & \cdots & \cdots & \mathrm{Sc}_{i_{i_{n} 2}}(s) \bar{S}_{i_{n^{n}} n}(s) \\
\vdots & \vdots & \ddots & \ddots & \vdots \\
\vdots & \vdots & \ddots & \ddots & \vdots \\
\mathrm{Sc}_{i_{1} n}(s) \bar{S}_{i_{1} 1}(s) & \mathrm{Sc}_{i_{2} n}(s) \bar{S}_{i_{2} 2}(s) & \cdots & \cdots & \mathrm{Sc}_{i_{i_{n} n}}(s) \bar{S}_{i_{n} n}(s)
\end{array}\right]
\end{aligned}
$$

Then, the comparison of the two sides of (12) yields (11) and

$$
\mathrm{DC}_{i j}(s)=\frac{1}{\operatorname{det} \mathbf{G}_{\mathrm{S}}(s)} \mathrm{Sc}_{i_{i j}} \bar{S}_{i, j}
$$

By the comparison of (11) and (13), one can obtain (10).

The inverted decoupler $\mathbf{G}_{\mathrm{IDC}}(s)$ (4) can be obtained from (10) and (11) by using (7a). Alternatively, it is possible to use the following result.

Proposition 3. Decoupler $\mathbf{G}_{\mathrm{IDC}}(s)$ (4) satisfies condition (8) if

$$
\operatorname{IDC}_{i j}(s)=\frac{S_{i_{j} j}}{S_{i_{i} i}}\left(1+\operatorname{IDC}_{i i}(s)\right), \quad i, j=1,2, \ldots, n ; i \neq j
$$

Moreover, if $\operatorname{IDC}_{i i}(s)=0$, then the ideal decoupling is obtained. Otherwise, to reach a selected $\overline{\mathbf{G}}_{S}(s)$, it must be set

$$
\operatorname{IDC}_{i i}(s)=\frac{1}{\operatorname{det} \overline{\mathbf{G}}_{\mathrm{S}}(s)} \overline{\mathrm{S}} \mathrm{c}_{i_{i} i} S_{i_{i} i}-1, \quad i=1,2, \ldots, n
$$

where $\overline{\mathrm{S}} \mathrm{c}_{i j}$ stands for the cofactor of $\bar{S}_{i j}$.

The proof is analogous to that of Proposition 2.

Remark 1. Note that for TITO systems, the ideal decoupling condition for the standard inverted decoupler (6) has two implicit solutions denoted as " $1-2$ " and " $2-1$ ", 46 for which it holds that

$$
\begin{aligned}
& \mathbf{G}_{\mathrm{Dd}, 1-2}(s)=\mathbf{I}, \mathbf{G}_{\mathrm{Do}, 2-1}(s)=-\mathbf{I}, \mathbf{G}_{\mathrm{Do}, 1-2}(s) \\
& \quad=-\mathbf{G}_{\mathrm{Dd}, 2-1}^{-1}(s)
\end{aligned}
$$

When substituting (16) into (7b), we have

$$
\mathbf{G}_{\mathrm{IDC}}(s)=-\mathbf{G}_{\mathrm{Do}, 1-2}(s)=\mathbf{G}_{\mathrm{Dd}, 2-1}^{-1}(s)
$$

The introduction of the additional feedback loop into the inverted decoupling subsystem (as in Figure 2b) leads to a transfer function matrix of the decoupler expressed by noninverted functions $\mathbf{G}_{\mathrm{Dd}, 2-1}(s)$ and $\mathbf{G}_{\mathrm{Do}, 2-1}(s){ }^{65}$

In contrast to the standard inverted decoupler, the proposed one provides the explicit, unambiguous formulae (10), (11), (14), and (15).

2.4. Measurable Disturbance Elimination. Whenever the feedback control system is affected by measurable disturbances, they can be compensated for by antidisturbance controllers. Assume that the disturbance $\mathbf{d}(t)$ acts on the plant as shown in Figure 1. The (absolute) invariance condition is expressed as

$$
\begin{aligned}
& \mathbf{G}_{\mathrm{DY}}(s)=0 \Leftrightarrow \mathbf{G}_{\mathrm{SD}}(s)-\mathbf{G}_{\mathrm{S}}(s) \mathbf{G}_{\mathrm{AC}}(s)=0 \Leftrightarrow \mathbf{G}_{\mathrm{AC}}(s) \\
& \quad=\mathbf{G}_{\mathrm{S}}^{-1}(s) \mathbf{G}_{\mathrm{SD}}(s)
\end{aligned}
$$

or, equivalently as

$$
\begin{aligned}
& \mathrm{AC}_{i j}=\frac{1}{\operatorname{det} \mathbf{G}_{\mathrm{S}}(s)} \sum_{k=1}^{n} \mathrm{Sc}_{k i} \mathrm{SD}_{k j}, \quad i=1,2, \ldots, n ; j=1,2, \\
& \quad . ., m, m \leq n
\end{aligned}
$$

2.5. Selected PID Tuning Rules. If an entire decoupling scheme is achieved, decoupling design results in the modified controlled system represented by a set of independent SISO transfer functions $\overline{\mathbf{G}}_{S}(s)$, where all nondominant crossinteractions are canceled. Then, the primary controllers $C_{i j}(s)$ in $\mathbf{G}_{\mathrm{C}}(s)$ are to be set such that $C_{i j}(s) \neq 0$ whenever $y_{j}-u_{i}$ is the dominant control pair, otherwise, $C_{i j}(s)=0$. The overview of three controller tuning methods used in our research follows.

2.5.1. Balanced Tuning Method. The leading idea of balanced tuning is to achieve the equality

$$
\int_{0}^{\infty} t|e(t)| \mathrm{d} t=T_{\mathrm{I}} \int_{0}^{\infty} t|\dot{e}(t)| \mathrm{d} t, \quad \dot{e}(t)=\frac{\mathrm{d} e(t)}{\mathrm{d} t}
$$

where $T_{\mathrm{I}}$ is the controller integral time constant. ${ }^{67}$ The determination of controller parameters is based on the first order plus time delay plant model

$$
G_{S}(s)=\frac{K}{T s+1} \mathrm{e}^{-\tau s}
$$

Assume the ideal continuous-time PID controller governed by the transfer function

$$
G_{\mathrm{C}}(s)=K_{\mathrm{P}}\left(1+\frac{1}{T_{\mathrm{I}} s}+T_{\mathrm{D}} s\right)
$$

where $K_{\mathrm{P}}$ and $T_{\mathrm{D}}$ are the controller gain and the derivative time constant, respectively. The setting rules read 


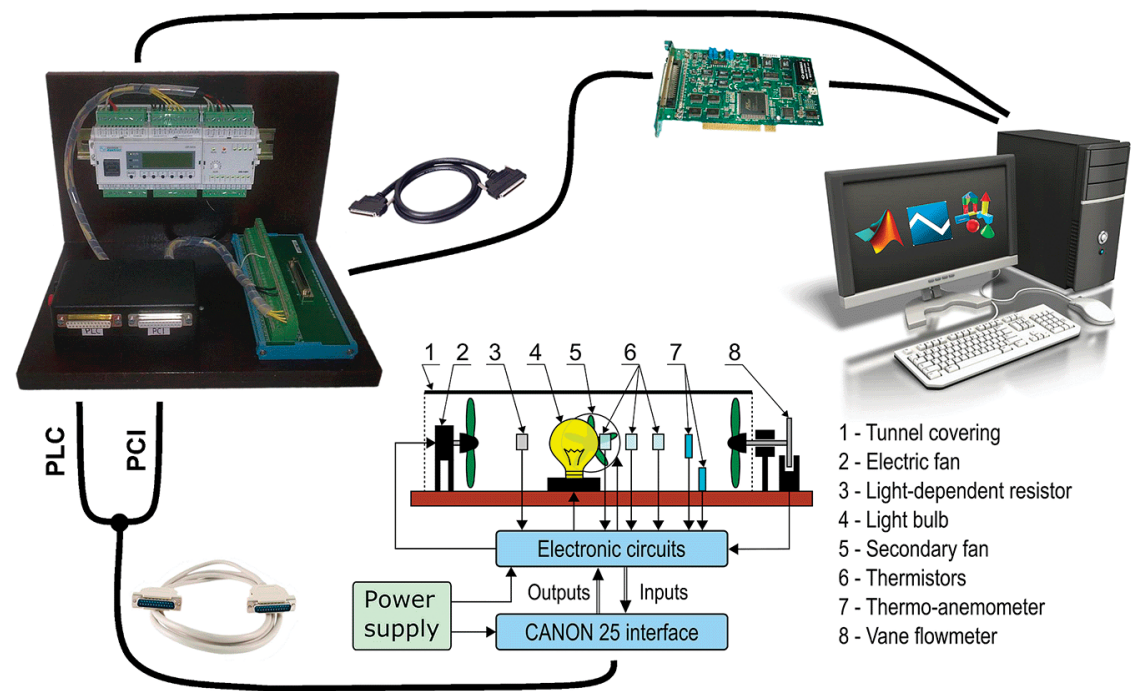

Figure 3. Hot-air tunnel laboratory model with interconnections.

$$
\begin{aligned}
& K_{\mathrm{P}}=\frac{1}{K}\left(1-\frac{2 \theta}{1+\sqrt{1+2 \theta^{2}}}\right) \\
& T_{\mathrm{I}}=\left(\frac{1+\sqrt{1+2 \theta^{2}}}{2}-\theta\right) T_{\mathrm{ar}} \\
& T_{\mathrm{D}} \leq \frac{T_{\mathrm{I}}}{4}
\end{aligned}
$$

where $\theta$ is the normalized delay and $T_{\text {ar }}$ stands for the average residence time

$$
\theta=\frac{\tau}{T_{\mathrm{ar}}}, \quad T_{\mathrm{ar}}=\tau+T
$$

2.5.2. Desired-Model Method. The method attempts to assign the desired reference-to-output transfer function based on the given model of the controlled plant. In this research, we apply the approach to the controlled model of the form

$$
G_{S}(s)=\frac{K}{\left(T_{1} s+1\right)\left(T_{2} s+1\right)}, \quad T_{1}>T_{2}
$$

for which the PID controller setting rules read ${ }^{68}$

$$
\begin{aligned}
& K_{\mathrm{P}}=\frac{2 T_{\mathrm{I}}}{K\left(2 T_{\mathrm{W}}+T_{\mathrm{s}}\right)} \\
& T_{\mathrm{I}}=T_{1}+T_{2}-T_{\mathrm{s}} \\
& T_{\mathrm{D}} \leq \frac{T_{1} T_{2}}{T_{1}+T_{2}}-\frac{T_{\mathrm{s}}}{4}
\end{aligned}
$$

where $T_{\mathrm{s}}$ means the sampling period (for continuous-time systems, $T_{\mathrm{s}}=0$ ) and $T_{\mathrm{W}}$ is the desired control system time constant that must satisfy the condition

$$
T_{\mathrm{s}}<\eta T_{\mathrm{W}}, \quad \eta<0.3
$$

2.5.3. Algebraic Polynomial Method. The method adopts the fractional formulation of transfer functions and signals in the control loop over the ring of polynomials. ${ }^{69,70}$ Control aims are to reach control system (Hurwitz) stability with given feedback pole loci and reference tracking. Let the controlled SISO plant model and the controller be expressed as follows, respectively,

$$
G_{\mathrm{S}}(s)=\frac{b(s)}{a(s)}, \quad G_{\mathrm{C}}(s)=\frac{q(s)}{p(s)}
$$

where $a(s), b(s), p(s), q(s)$ are coprime polynomials satisfying $\operatorname{deg} a(s)<\operatorname{deg} b(s), \operatorname{deg} p(s)<\operatorname{deg} q(s)$. Let the single-variable reference signal have polynomial fractional representation as well

$$
r(s)=\frac{h_{\mathrm{r}}(s)}{f_{\mathrm{r}}(s)}
$$

Then, the feedback control system in Figure 1 is Hurwitz stable if and only if

$$
a(s) p(s)+b(s) q(s)=\bar{\Delta}(s)
$$

where $\bar{\Delta}(s)=\prod_{i=1}^{\operatorname{deg} \bar{\Delta}(s)}\left(s-\bar{s}_{i}\right)$ stands for the desired characteristic polynomial governed by desired poles $\overline{s_{i}} \in \mathbb{C}^{-}$. The reference signal is tracked if, moreover, $f_{\mathrm{r}}(s)$ divides $a(s)$ $p(s)$, i.e., the expression

$$
\frac{a(s) p(s)}{f_{\mathrm{r}}(s)}
$$

is a polynomial. ${ }^{69,70}$

2.6. Selected Identification Methods and Tools. Parameters of a selected plant model are primarily estimated via System Identification Toolbox with Process Models and Linear Parametric Models interfaces in Matlab. ${ }^{71}$ The obtained results are verified using the standard LMS method (which is the most used stochastic regressive identification procedure) for the ARX MISO model. The identification procedure is used for every single output in the case of a multioutput model. As it is a standard procedure, its description is omitted, and further details can be found, e.g., in Bobál et al. ${ }^{72}$

2.7. Feedback Robust Stability Overview. Real-world systems face their imprecise modeling, nonlinearities, highorder behavior, disturbances, and many other effects that act on the system and cause their model imperfections and uncertainties. $^{70}$ Stability represents one of the fundamental requirements of the designed control system. The endeavor to 
satisfy specific feedback stability under the uncertainties gives rise to robust stability. ${ }^{73}$ We do let recall basic facts about robust control feedback stability when the controlled MIMO plant is assumed to be perturbed under multiplicative (input) uncertainties, i.e.,

$$
\hat{\mathbf{G}}_{\mathrm{S}}(s)=\mathbf{G}_{\mathrm{S}}(s)[\mathbf{I}+w \boldsymbol{\Delta}(s)]
$$

where $\mathbf{G}_{\mathrm{S}}(s)$ represents the nominal plant model as in (1), $\boldsymbol{\Delta}(s)$ means the full complex perturbation matrix satisfying $\|\boldsymbol{\Delta}(s)\|_{\infty} \leq 1 \Leftrightarrow \sigma(\boldsymbol{\Delta}(j \omega)) \leq 1,0 \leq \omega<\infty$ (where $\sigma(\cdot)$ stands for the spectral radius) and $w$ is the (scalar) weight, for which it holds that

$$
w \leq\left\|\mathbf{G}_{\mathrm{S}}^{-1}(s)\left[\hat{\mathbf{G}}_{\mathrm{S}}(s)-\mathbf{G}_{\mathrm{S}}(s)\right]\right\|_{\infty}
$$

The control feedback system (2) and (3) is robustly stable if and only if if,74 $^{73}$

$$
\left\|\mathbf{G}_{\mathrm{D}}(s) \mathbf{G}_{\mathrm{R}}(s) \cdot\left[\mathbf{I}+\mathbf{G}_{\mathrm{S}}(s) \mathbf{G}_{\mathrm{D}}(s) \mathbf{G}_{\mathrm{C}}(s)\right]^{-1} \mathbf{G}_{\mathrm{S}}(s) w\right\|_{\infty}<1
$$

\section{EXPERIMENTAL SETUP}

3.1. Hot-Air Tunnel System Description. The controlled hot-air tunnel is composed of a light bulb, an electric fan, and sensors to measure temperature, brightness, and air flow (see Figure 3). The bulb and the fan serve as heat and air-flow sources, respectively. The secondary fan, placed from the backside, acts as a disturbance source. There are three thermistors for the temperature measurement with the operation range $[-50,150]{ }^{\circ} \mathrm{C}$ in the appliance. Namely, temperature values on the bulb surface, nearby the bulb, and inside the back part of the tunnel are measured. The brightness can be measured via a light-dependent resistor (photoresistor).

The power (voltage) supply is supplied by the $230 \mathrm{~V} / 50 \mathrm{~Hz}$ line voltage. The electronic circuit voltage output is within the range $\pm 15 \mathrm{~V}$. The 25-pin CANON interface connects the supply and the electronic circuits with a control and supervising subsystem with inputs and outputs of $[0,10] \mathrm{V}$, except for the secondary fan, the output voltage range of which is $[0,12] \mathrm{V}$. One can either connect the model with a PLC or a PC equipped with a PCI $1711 \mathrm{U}$ card (see Figure 3).

The auxiliary unit interconnecting the tunnel model with a supervisory and control subsystem can be seen on the lefthand side of the figure, the functioning of which is described below.

3.2. PLC and PCl Card Specification. 3.2.1. PCl Card. The PCI $17 \mathrm{xx}$ series cards ${ }^{75}$ are data acquisition and control industrial devices by Advantech. The PCI $1711 \mathrm{U}$ bus is used to handle the communication between the tunnel model (the auxiliary unit, more precisely) and the Matlab/Simulink environment in the PC that reads and writes data (see Figure 4). It represents a multifunctional card incorporating 16 analog inputs (AI), 2 analog outputs (AO), 16 digital inputs (DI), 16 digital outputs (DO), and a timer/counter. AI and

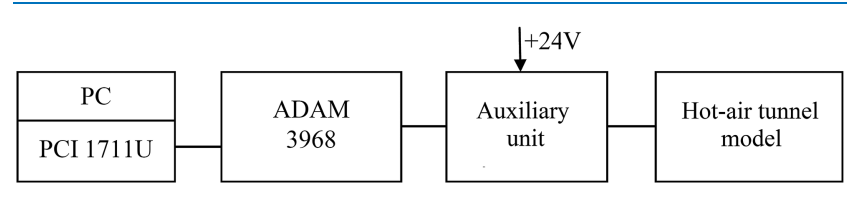

Figure 4. The PCI card to the tunnel connection scheme.
AO contain a 12-bit converter at the maximum frequency of $100 \mathrm{kHz}$, and they have a voltage range of $\pm 10 \mathrm{~V}$. AI utilizes a buffer for continuous data reading. DI and DO are compatible with the transistor-transistor logic (TTL).

The card is equipped with a 68-pin input/output connector that is linked to an ADAM-3968 terminal plate and a DIN rail. The terminal plate is connected to an auxiliary unit that is powered by $24 \mathrm{~V} \mathrm{DC}$ and controls the secondary fan at $[0$, $12] \mathrm{V}$ via a DO from the PCI card. It is interconnected with the hot-air tunnel using a cable and a CANON 25 interface.

The Real-Time Windows Target library is used for communication. The sampling period $\left(T_{s}\right)$, the channel number, and input-output signal parameters ought to be set for used function blocks included in the library. In different sampling periods of the used blocks, a rate transition block must be placed between the blocks to hold the samples between the time instances (see Figure 5 that displays a solution example). Then, the model must be built and compiled in $\mathrm{C}$ language before its running.

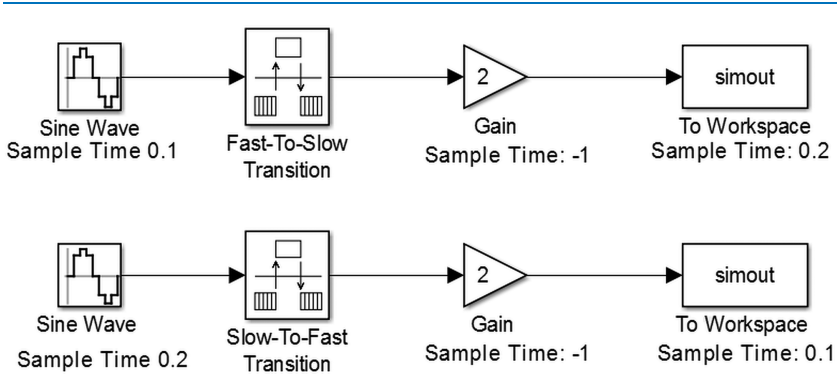

Figure 5. Communication between function blocks with different sampling periods.

3.2.2. PLC Unit. The use of PLC by Teco represents another possibility of the model control. The basic CP-1015 module and OS-1401 extending module are applied. In the basic module, two 12-bit $\mathrm{AO}$ and four 12-bit AI are utilized. Since it contains only two AO, the secondary fan has to be controlled via a DI. The extending module incorporates 12 TTL outputs that have a common terminal powered by $12 \mathrm{~V}$ from the auxiliary unit (as for the PCI card). A scheme of the interconnection of the hot-air tunnel model and the PLC is displayed in Figure 6.

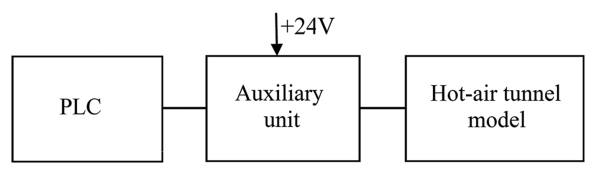

Figure 6. The PLC to the tunnel connection scheme.

It is necessary to connect TCL2+ and TCL2- outputs to get the correct communication between the basic and the extending modules, and the latter one has to be terminated by using TXN 10290 . The auxiliary unit serves as the $24 \mathrm{~V}$ power source for the PLC.

\section{RESULTS AND DISCUSSION}

The three most critical dynamic parameters of a tunnel are the length, temperature, and hot-air speed. ${ }^{10}$ As already introduced, the considered laboratory hot-air tunnel has to be taken as a multivariable system since the air flow generated by the (main) electric fan influences not only the flow rate but 

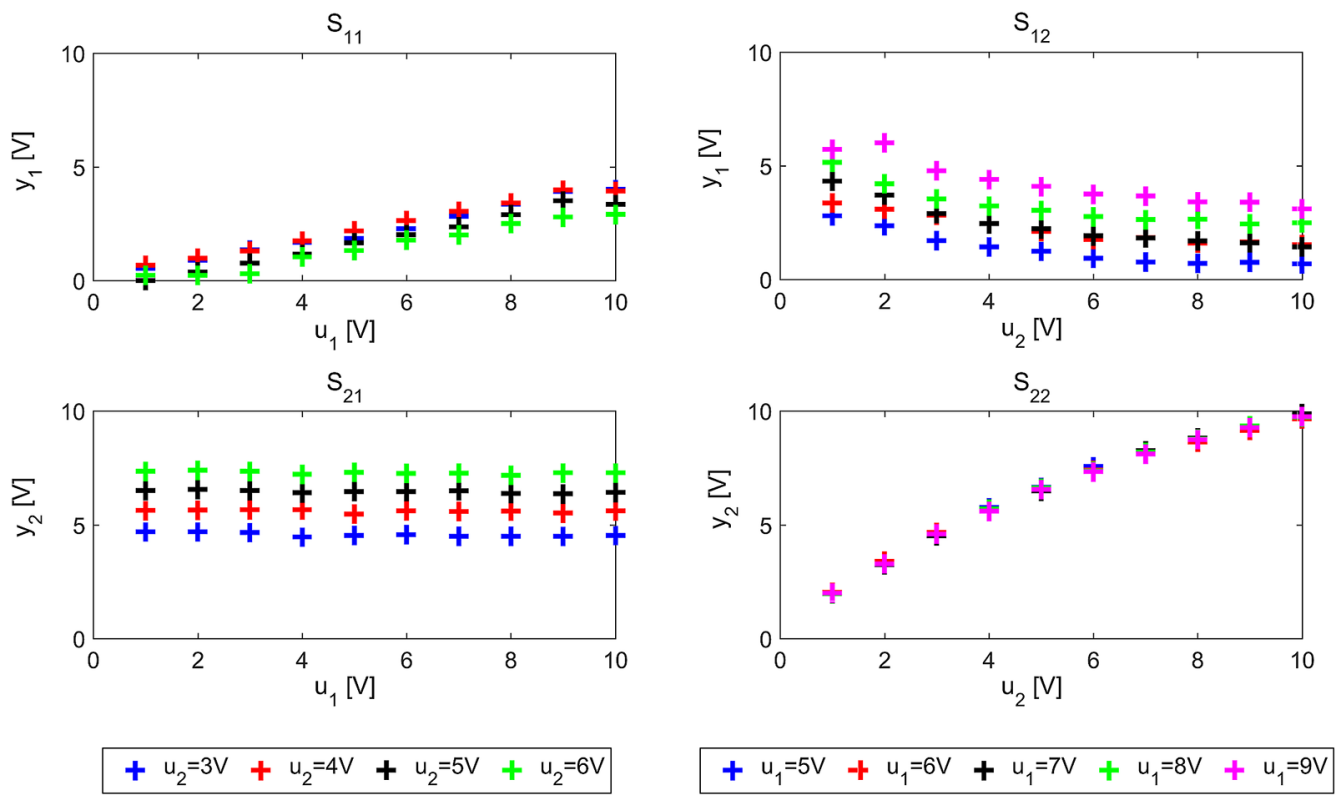

Figure 7. Static characteristics of $\mathbf{G}_{S}(s)$ measured by the PLC when $d=d^{*}=7$.
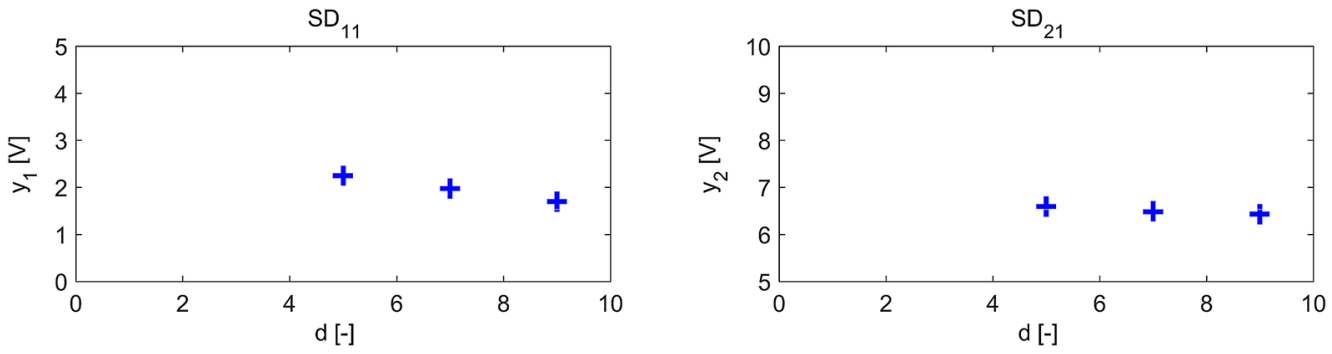

Figure 8. Static characteristics of $\mathbf{G}_{\mathrm{SD}}(s)$ measured by the PLC when $\mathbf{u}=\mathbf{u}^{*}=[6,5]^{\mathrm{T}}$.
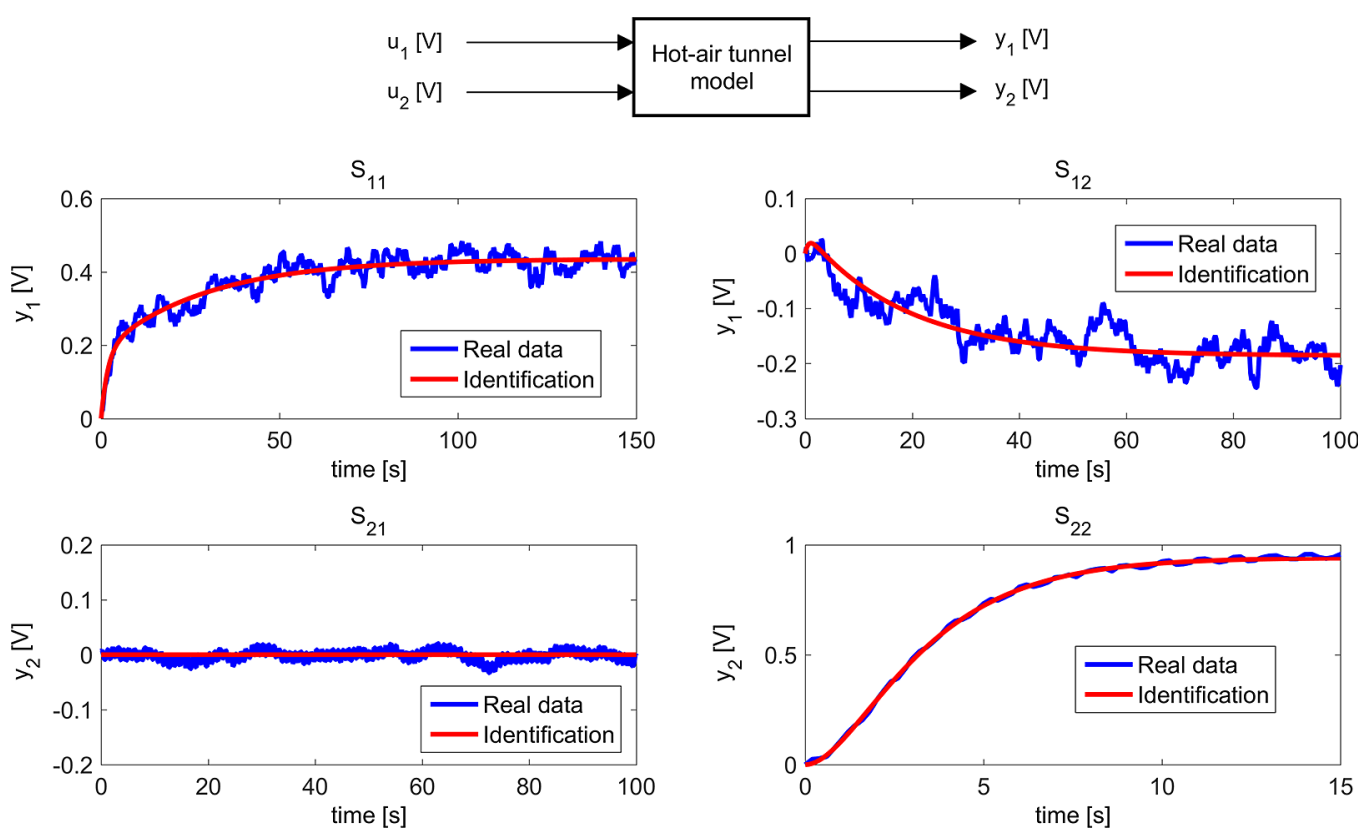

Figure 9. Measured and identified unit step responses of $\mathbf{G}_{S}(s)$ using the PLC and Identification Toolbox at the operating point $\mathbf{u}^{*}=[6,5]^{\mathrm{T}}, d^{*}$ $=7$.

also the temperature inside the tunnel. The flow caused by the secondary fan can be considered as a disturbance that affects both the outputs as well.
The bulb input voltage and the fan input voltage are taken as manipulated input variables $u_{1}(t)$ and $u_{2}(t)$, respectively.

The disturbance $d(t)$ (within the dimensionless range $d_{1}=d$ 

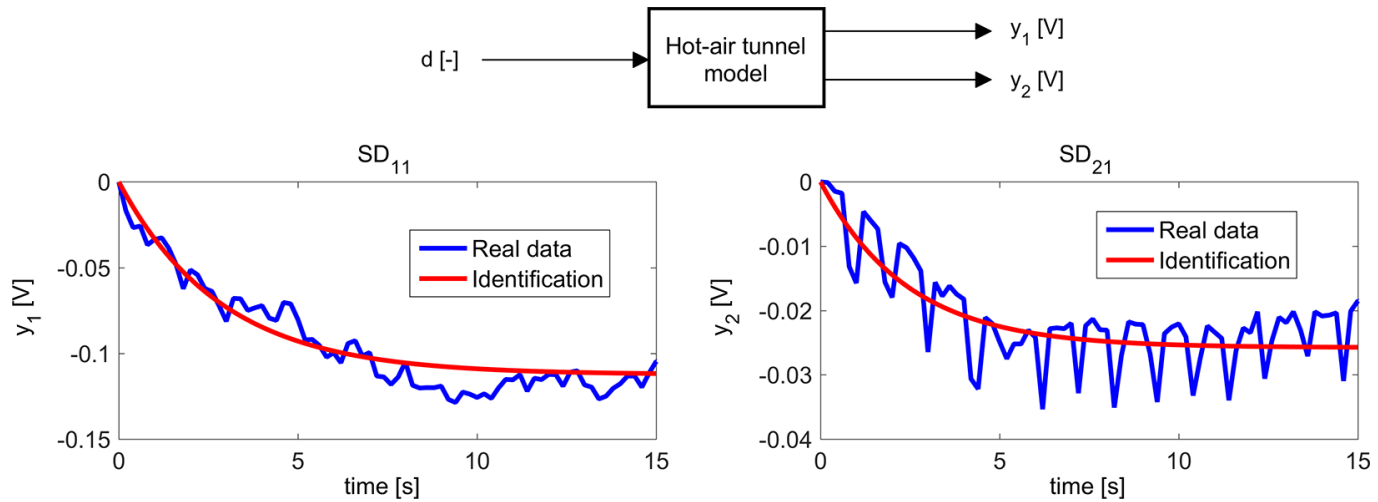

Figure 10. Measured and identified unit step responses of $\mathbf{G}_{\mathrm{SD}}(s)$ using the PLC and Identification Toolbox at the operating point $\mathbf{u}^{*}=[6,5]^{\mathrm{T}}$, $d^{*}=7$.

$\in[0,10])$ is measured as the input voltage $d_{\mathrm{V}}$ to the secondary fan, for which the following correspondence holds: $d_{\mathrm{V}}=1.2 d \mathrm{~V}$. It is worth noting that the secondary fan does not turn until $d_{\mathrm{V}}=5 \mathrm{~V}$.

The output (controlled) variable $y_{1}(t)$ is the thermistor voltage, and $y_{2}(t)$ is the vane flowmeter voltage. Note that the leftmost (see Figure 3) thermistor is taken for $y_{1}(t)$, as it exhibits the steepest slope of the static characteristic.

4.1. Identification Experiments. Identification experiments are done by using the PLC and via a PC equipped with the PCI card separately. Besides, in each of the cases, static and dynamic characteristics are measured. The shape of dynamic characteristics yields the selection of a suitable generalized model form. That is, the induction principle rather than the deduction one is applied. Then, the selected model parameters are to be estimated.

4.1.1. Model Parameter Identification Using PLC. For static characteristic measurements, one of the manipulated inputs varies within the range $[1,10] \mathrm{V}$ while another is constant (at the operating point). The measurement is made repeatedly for different constant input values. Static characteristics of $S_{11}(s)$ and $S_{12}(s)$ imply not only that the increase of $u_{2}$ causes the temperature decrease inside the tunnel but also that if $u_{1}$ is low (i.e., approx. $u_{1} \in[1,3] \mathrm{V}, u_{2}=6 \mathrm{~V}$ ), the maximum cooling is obtained (see Figure 7). As expected, the air-flow rate is affected only by $u_{2}$, see static characteristics of $S_{21}(s)$ and $S_{22}(s)$. Two distinct linear sections (i.e., $u_{2} \in[1,4]$ $\mathrm{V}$ and $\left.u_{2} \in[4,10] \mathrm{V}\right)$ compose the static characteristic of $S_{12}(s)$. Note that $d=d^{*}=7$ for Figure 7 .

Regarding the disturbance, static characteristics of $\mathrm{SD}_{11}(s)$ and $\mathrm{SD}_{21}(s)$ (see Figure 8) are measured only for $d>5$ due to the minimum required switching voltage of the secondary fan (i.e., $d_{\mathrm{V}} \geq 5 \mathrm{~V}$ ) while the operating point $\mathbf{u}^{*}=[6,5]^{\mathrm{T}} \mathrm{V}$ is taken.

The same operating point is selected for step response measurements of $\mathbf{G}_{\mathrm{S}}(s)$ (Figure 9). As nonunit constant input changes of $\Delta u_{1}=\Delta u_{2}=2 \mathrm{~V}$ (i.e., $\mathbf{u}=[8,5]^{\mathrm{T}} \mathrm{V}$ and $\mathbf{u}=[6$, $7]^{\mathrm{T}} \mathrm{V}$ ) are taken for the measurements, the figure includes normalized (rescaled) unit step responses (i.e., for the input step change levels of $1 \mathrm{~V}$ ). The model is assumed to be linear in the vicinity of $\mathbf{u}^{*}$ (see Figures 7 and 8). In Figure 9, a schematic sketch elucidating the corresponding input-output pairs is given as well.

The responses are of relatively complex dynamics (see Figure 9); hence, the second-order model (34) with finite zeros is selected for $S_{11}(s)$ and $S_{12}(s)$ :

$$
G_{\mathrm{S}}(s)=\frac{K\left(T_{\mathrm{N}} s+1\right)}{\left(T_{1} s+1\right)\left(T_{2} s+1\right)}, \quad T_{1}>T_{2}
$$

As $\Delta u_{1}$ does not excite $y_{2}(t)$ significantly, relation $S_{21}(s)$ is neglected in the model, which agrees with the physical properties of the tunnel. The response of $y_{2}(t)$ to $\Delta u_{2}=1 \mathrm{~V}$ has the standard shape of the second-order overdamped system without an overshoot; therefore, (24) is selected for the $S_{22}(s)$ model. The identical observation also holds for $\mathrm{SD}_{11}(s)$ and $\mathrm{SD}_{21}(s)$ (see Figure 10 ). The disturbance step change of $\Delta d=2$ (i.e., $d=9$ ) is taken for measurements when $\mathbf{u}=\mathbf{u}^{*}$; yet the displayed responses are normalized to the unit step input as the model is supposed to be linear in the neighborhood of the operating point again (see Figure 8).

The particular transfer function parameters are primarily identified using the Identification Toolbox (function ident), resulting in (35).

$$
\begin{aligned}
\mathbf{G}_{\mathrm{S}}(s) & =\left[\begin{array}{cc}
\frac{0.435(13.416 s+1)}{(29.173 s+1)(1.877 s+1)} & \frac{-0.186(-3.018 s+1)}{(18.597 s+1)(0.623 s+1)} \\
0 & \frac{0.941}{(2.162 s+1)(1.425 s+1)}
\end{array}\right] \\
\mathbf{G}_{\mathrm{SD}}(s) & =\left[\begin{array}{c}
\frac{-0.112}{2.855 s+1} \\
\frac{-0.0257}{2.429 s+1}
\end{array}\right]
\end{aligned}
$$

We do let attempt to use the LMS identification procedure for the second-order ARX model for the comparison as well, yielding

$$
\begin{aligned}
& \mathbf{G}_{\mathrm{S}}(s)=\left[\begin{array}{cc}
\frac{0.441(15.558 s+1)}{(31.201 s+1)(1.756 s+1)} & \frac{-0.198(-3.227 s+1)}{(19.239 s+1)(0.589 s+1)} \\
0 & \frac{0.964(0.051 s+1)}{(2.209 s+1)(1.277 s+1)}
\end{array}\right] \\
& \mathbf{G}_{\mathrm{SD}}(s)=\left[\begin{array}{c}
\frac{-0.105(0.041 s+1)}{(2.659 s+1)(0.063 s+1)} \\
\frac{-0.0189(0.081 s+1)}{(2.522 s+1)(0.066 s+1)}
\end{array}\right]
\end{aligned}
$$

Models (35) and (36) are apparently very close to each other, measured by the time constant values and static gains. Note that the transformation of the discrete-time ARX model 

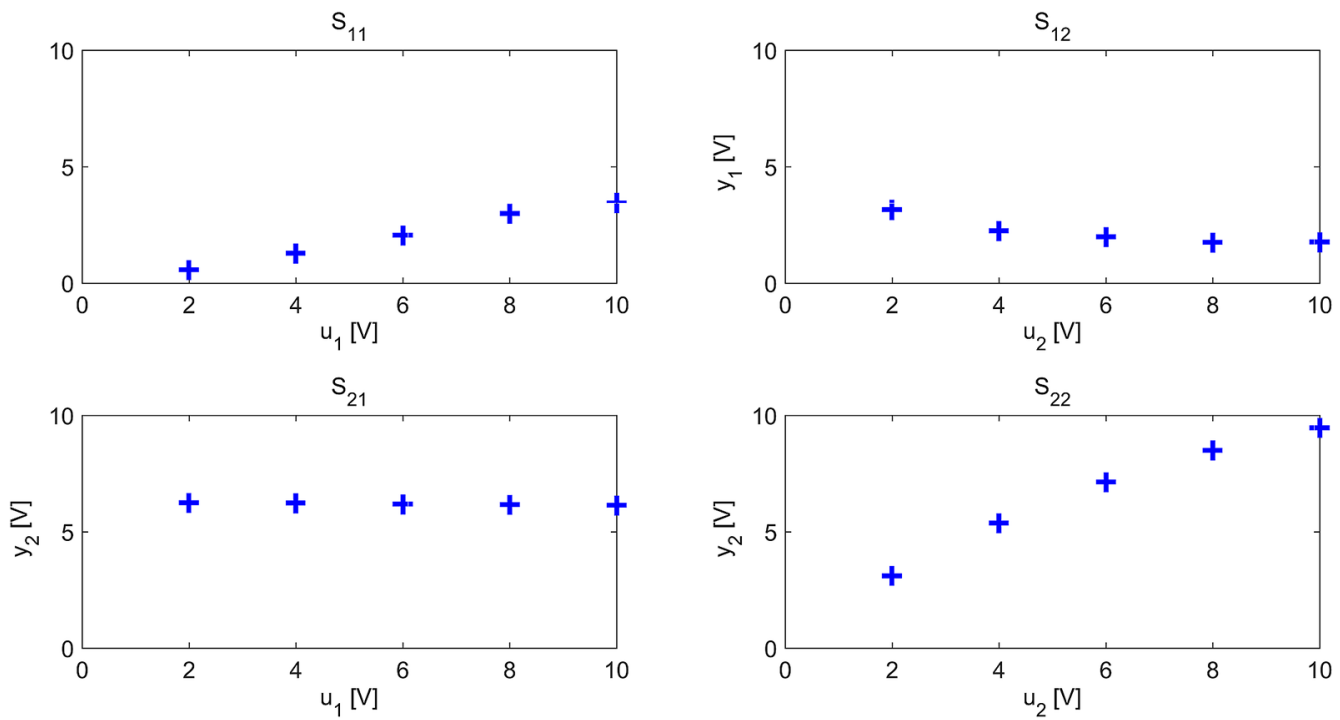

Figure 11. Static characteristics of $\mathbf{G}_{S}(s)$ measured by the PCI card when $d=d^{*}=7$.
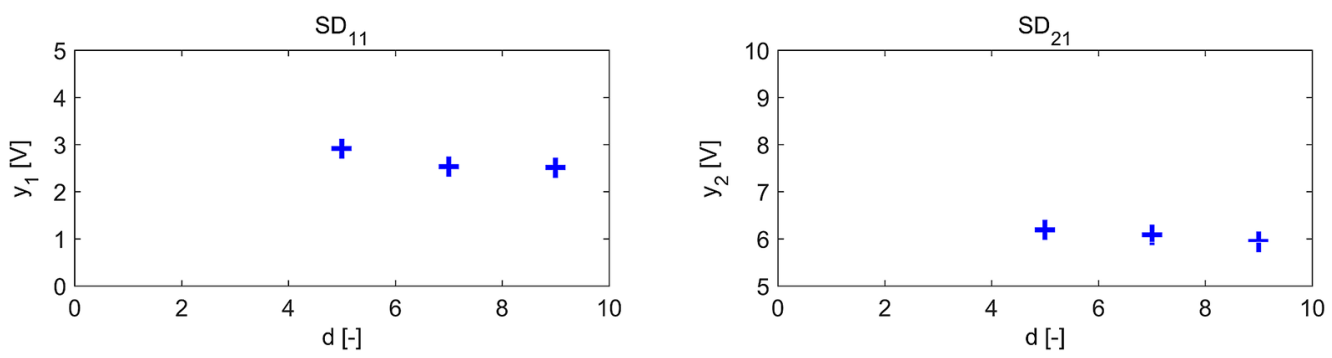

Figure 12. Static characteristics of $\mathrm{G}_{\mathrm{SD}}(s)$ measured by the PCI card when $\mathrm{u}=\mathrm{u}^{*}=[6,5]^{\mathrm{T}}$.
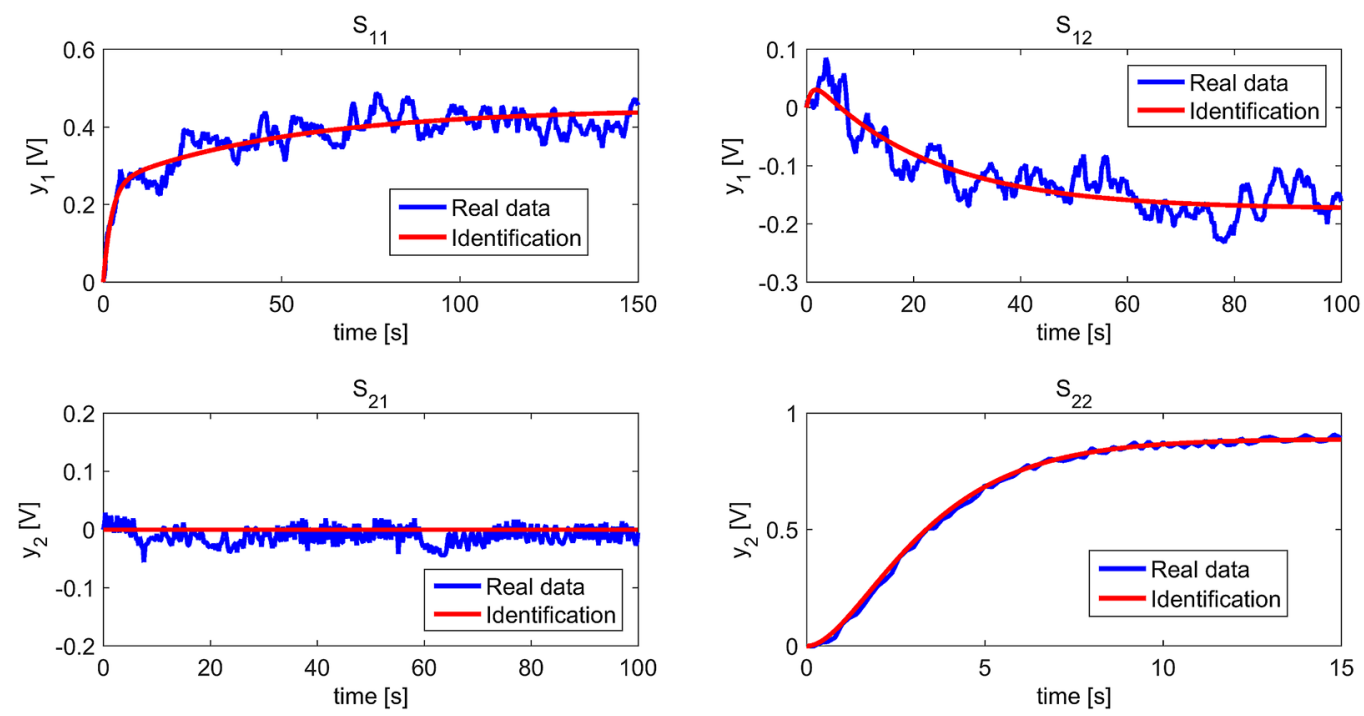

Figure 13. Measured and identified step responses of $\mathbf{G}_{S}(s)$ using the PCI card and Identification Toolbox at the operating point $\mathbf{u}^{*}=[6,5]^{\mathrm{T}}, d^{*}$ $=7$.

to continuous-time one (34) is made via standard the $d 2 c$ Matlab function with the zero-order hold and $T_{\mathrm{s}}=0.2$.

4.1.2. Model Parameter Identification Using PCI Card and $P C$. Static characteristics and step responses are also measured by the connection of the tunnel model and a PC equipped with the PCI card (Figures 11-14). Then, the Matlab toolbox's identification procedure is made again to verify the connection correctness and the closeness of measured properties (with respect to the PLC test). The operating point and input step changes are the same as for the PLC case, i.e., $\mathbf{u}^{*}=[6,5]^{\mathrm{T}} \mathrm{V}, d=7, \Delta u_{1}=\Delta u_{2}=1 \mathrm{~V}, \Delta d=$ 1. For instance, the static characteristic point for $S_{11}(s)$ in Figure 11 is obtained for fixed $u_{2}=5 \mathrm{~V}, d=7$ while $u_{1}$ ranges from 2 to $10 \mathrm{~V}$. Similarly, the upper-left response in Figure 13 is the dynamic response to the step change of $u_{1}(t)$ from 6 to $7 \mathrm{~V}$ while $u_{2}=5 \mathrm{~V}, d=7$. 

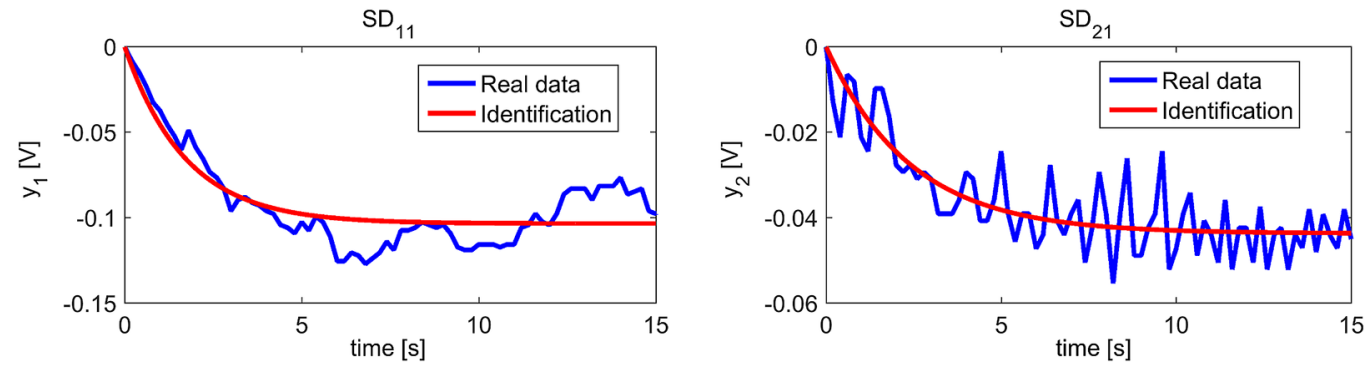

Figure 14. Measured and identified step responses of $\mathbf{G}_{\mathrm{SD}}(s)$ using the PCI card and Identification Toolbox at the operating point $\mathbf{u}^{*}=[6,5]^{\mathrm{T}}$, $d^{*}=7$.

The resulting models are as follows

$$
\begin{aligned}
\mathbf{G}_{\mathrm{S}}(s) & =\left[\begin{array}{cc}
\frac{0.447(29.702 s+1)}{(50.892 s+1)(2.266 s+1)} & \frac{-0.174 \cdot(-6.088 s+1)}{(22.271 s+1)(0.963 s+1)} \\
0 & \frac{0.889}{(2.068 s+1)(1.507 s+1)}
\end{array}\right] \\
\mathbf{G}_{\mathrm{SD}}(s) & =\left[\begin{array}{c}
\frac{-0.103}{1.716 s+1} \\
\frac{-0.043}{2.416 s+1}
\end{array}\right]
\end{aligned}
$$

It can be observed that results (37) are very close to those in (35) and (36).

Remark 2. The dynamic responses for the model parameter identification are not made for step-down input changes as our intention is to get a unique simple linear model for practical experiments rather than perform a more rigorous identification procedure. Indeed, the controlled process can be considered as linear in the vicinity of the operating point (Figures 7, 8, 11, and 12).

4.2. Controllers Design and Tuning for the Tunnel Model. Control feedback stability, full decoupling, and absolute invariance to the measurable disturbance are three main control aims. Process model (35) is assumed, for which the control system design and tuning follow, based on the calculations given in Sections 2.3-2.5. The primary controller $C_{11}(s)$ (for the temperature control) is designed using the polynomial (algebraic) method, while the balanced tuning method and the desired-model method are applied to design $C_{22}(s)$ (for the air-flow rate control).

4.2.1. Decoupler Design. Any method overviewed in Section 1 prior to a control loop decoupling and decentralized controller design should be made by selecting suitable or even the optimal control pairs. However, since $S_{21}=0$, the only admissible control pairs read $y_{1}-u_{1}$ and $y_{2}-u_{2}$. Following the ideal full decoupling condition (9), one has $\mathbf{M}=\mathbf{I}$ and

$$
\overline{\mathbf{G}}_{\mathrm{S}}(s)=\left[\begin{array}{cc}
\frac{0.435(13.416 s+1)}{(29.173 s+1)(1.877 s+1)} & 0 \\
0 & \frac{0.941}{(2.162 s+1)(1.425 s+1)}
\end{array}\right]
$$

The proposed inverted decoupler is obtained via (14) for zero diagonal elements of $\mathbf{G}_{\mathrm{IDC}}(s)$, giving rise to

$$
\begin{aligned}
& \mathbf{G}_{\mathrm{IDC}}(s) \\
& \quad=\left[\begin{array}{lc}
0 & \frac{0.4546 s^{3}+0.1071 s^{2}-7.711 \times 10^{-2} s-2.751 \times 10^{-3}}{s^{3}+1.7334 s^{2}+0.21 s+6.433 \times 10^{-3}} \\
0 & 0
\end{array}\right]
\end{aligned}
$$

Transformation (7a), or equivalently, formulae (10) and (11), yields the noninverted decoupler (5) transfer function matrix

$$
\mathbf{G}_{\mathrm{DC}}(s)=\left[\begin{array}{cc}
1 & \frac{-0.4546 s^{3}-0.1071 s^{2}+7.711 \times 10^{-2} s+2.751 \times 10^{-3}}{s^{3}+1.7334 s^{2}+0.21+6.433 \times 10^{-3}} \\
0 & 1
\end{array}\right]
$$

4.2.2. Antidisturbance Controller Design. The antidisturbance controllers to get absolute invariance to the measurable disturbance are set via (18), giving rise to the transfer function vector

$$
\mathbf{G}_{\mathrm{AC}}(s)=\left[\begin{array}{c}
\left(9.372 \times 10^{-3} s^{6}-0.5354 s^{5}-1.332 s^{4}\right. \\
-0.8276 s^{3}-0.1627 s^{2}-1.002 \times 10^{-2} s \\
\left.-1.839 \times 10^{-4}\right) /\left(s^{5}+2.261 s^{4}+1.191 s^{3}\right. \\
\left.+0.2321 s^{2}+1.73 \times 10^{-2} s+4.26 \times 10^{-4}\right) \\
\left(-2.022 \times 10^{-2} s^{2}-2.353 \times 10^{-2} s\right. \\
\left.-6.559 \times 10^{-3}\right) /(s+0.205)
\end{array}\right]
$$

Although entries of (41) are not feasible in terms of the $s$ domain, they can be realized in the discrete-time domain by suitable (feasible) discretization of the derivative (or proportional-derivative) subsystem of (41). The reader is referred to Section 4.3 for more details.

4.2.3. Balanced Tuning Method. Let us use a simple approximation of $\bar{S}_{22}(s)$ by model (20) via ${ }^{68}$

$$
\bar{S}_{22}(s)=\frac{0.941}{(2.162 s+1)(1.425 s+1)} \approx \frac{0.941}{2.162 s+1} \mathrm{e}^{-1.425 s}
$$

Rules (22) and (23) yield

$$
C_{22}(s)=0.656\left(1+\frac{1}{2.426 s}+0.606 s\right)
$$

4.2.4. Desired-Model Method. Consider nonapproximated $\bar{S}_{22}(s)$ as in (42). With respect to the step response of $S_{22}(s)$ (see Figures 9 and 13), let $T_{\mathrm{W}}=5 \mathrm{~s}$. Then, design rules (25) for $T_{\mathrm{s}}=0$ give rise to

$$
C_{22}(s)=0.762\left(1+\frac{1}{3.587 s}+0.859 s\right)
$$



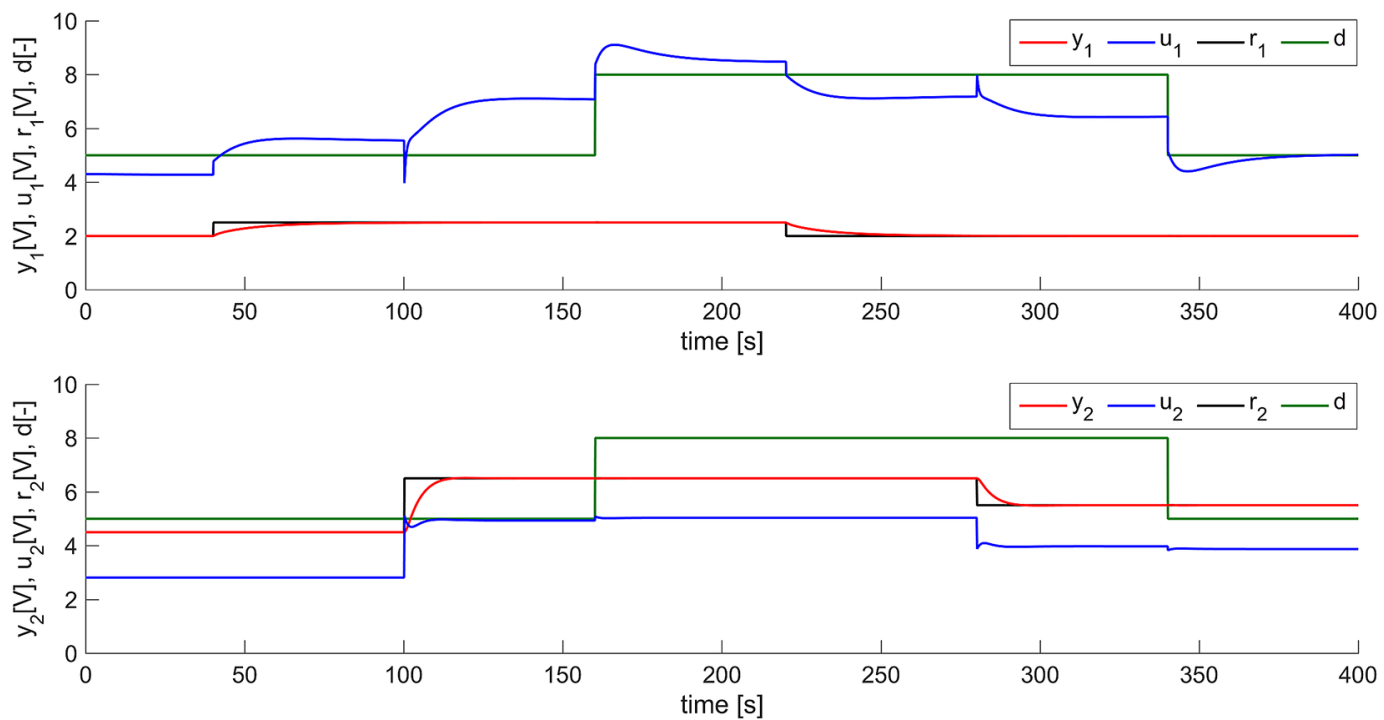

Figure 15. Simulated continuous-time control responses when using decoupling and antidisturbance controllers and $C_{22}(s)$ being set by the balanced tuning method.

4.2.5. Algebraic Polynomial Method. From (35) and (38), we have

$$
\bar{S}_{11}(s)=\frac{0.1067 s+7.953 \times 10^{-3}}{s^{2}+0.5668 s+1.825 \times 10^{-2}}=\frac{b(s)}{a(s)}
$$

We do let assume a step-wise reference function, i.e., $f_{\mathrm{r}}(s)=$ $s$. The following polynomial degrees are suggested to ensure controller feasibility and to get a unique solution of (29):

$$
\begin{aligned}
& \operatorname{deg} q(s)=\operatorname{deg} a(s)+\operatorname{deg} f_{\mathrm{r}}(s)-1=2 \\
& \operatorname{deg} \tilde{p}(s)=\operatorname{deg} a(s)-1=1 \\
& \operatorname{deg} \bar{\Delta}(s)=2 \operatorname{deg} a(s)+\operatorname{deg} f_{\mathrm{r}}(s)-1=1
\end{aligned}
$$

where $p(s)=\tilde{p}(s) f_{\mathrm{r}}(s)$. As it is a suitable choice to keep the closed-loop poles $\left(\bar{s}_{i}, i=1,2,3,4\right)$ nearby those of the controlled process, ${ }^{70}$ the following setting is made: $\bar{s}_{1}=\bar{s}_{2}$ $-0.05, \bar{s}_{3}=-0.1, \bar{s}_{4}=-0.7$. By substituting this option and also results of (46) into (29), the stabilizing formula yields

$$
C_{11}(s)=\frac{2.359 s^{2}+0.6493 s+2.2 \times 10^{-2}}{s^{2}+8.15 \times 10^{-2} s}
$$

which has a feasible PID structure. The controller feasibility means that the relative order of (47) is non-negative; or equivalently, the derivative term of this PID controller undergoes a low-pass filter.

4.3. Controller Discretization. To implement controllers (39), (41), (43), (44), and (47) by the PLC and the PCI card, their continuous-time formulations are to be translated into suitable discrete-time forms. Ideal PID controllers of type (21) are discretized via a backward difference scheme

$$
K_{\mathrm{P}}\left(1+\frac{1}{T_{\mathrm{I}} s}+T_{\mathrm{D}} s\right) \approx K_{\mathrm{P}}\left(1+\frac{T_{\mathrm{s}}}{T_{\mathrm{I}}} \frac{z}{z-1}+\frac{T_{\mathrm{D}}}{T_{\mathrm{s}}} \frac{z-1}{z}\right)=\frac{u(z)}{e(z)}
$$

where $z$ stands for the $z$-transform variable. Formula (48) corresponds to the following discrete-time realization:

$$
\begin{aligned}
u(k)= & u(k-1)+K_{\mathrm{P}}\left(1+\frac{T_{\mathrm{s}}}{T_{\mathrm{I}}}+\frac{T_{\mathrm{D}}}{T_{\mathrm{s}}}\right) e(k) \\
& -K_{\mathrm{P}}\left(1+2 \frac{T_{\mathrm{D}}}{T_{\mathrm{s}}}\right) e(k-1)+K_{\mathrm{P}} \frac{T_{\mathrm{D}}}{T_{\mathrm{s}}} e(k-2)
\end{aligned}
$$

where $u_{i}(k):=u_{i}\left(t_{k}\right), e(k):=e\left(t_{k}\right), t_{k+1}=t_{k}+T_{s}$.

Feasible PID controller (44) is subject to the feedforward Euler filter method as

$$
\begin{aligned}
& K_{\mathrm{P}}\left(1+\frac{1}{T_{\mathrm{I}}}+T_{\mathrm{D}} \frac{N s}{s+N}\right) \approx K_{\mathrm{P}}\left(1+\frac{T_{\mathrm{s}}}{T_{\mathrm{I}}} \frac{z}{z-1}+T_{\mathrm{D}} \frac{N \frac{z-1}{T_{\mathrm{s}}}}{T_{\mathrm{s}}}+N\right) \\
& =K_{\mathrm{P}}\left(1+\frac{T_{\mathrm{s}}}{T_{\mathrm{I}}} \frac{z}{z-1}+T_{\mathrm{D}} \frac{N}{1+\frac{N T_{\mathrm{s}}}{z-1}}\right)
\end{aligned}
$$

Antidisturbance controllers (41) are expressed by the parallel combination of the ideal PD controller and a strongly feasible subsystem $\widetilde{\mathrm{AC}}_{i 1}(s), i=1,2$ :

$$
\mathrm{AC}_{i 1}(s)=K_{\mathrm{P}}\left(1+T_{\mathrm{D}} s\right)+\widetilde{\mathrm{AC}}_{i 1}(s)
$$

Then, the PD part undergoes rule (48) and $\widetilde{\mathrm{AC}}_{i 1}(s)$ is subject to the Tustin approximation

$$
s \approx \frac{2}{T_{\mathrm{s}}} \frac{z-1}{z+1}
$$

Rule (52) is used to discretize controllers (39) and (40). Note that the sampling period is set to $T_{\mathrm{s}}=1 \mathrm{~s}$ in the control system.

4.4. Simulated Control Responses. Various simulation comparative tests are made in the Matlab/Simulink environment. Namely, the use of continuous-time controllers versus their discrete-time formulations is tested. The beneficial effect of decouplers and antidisturbance controllers is demonstrated by comparing the simple feedback control system. Finally, the primary controller $C_{22}(s)$ is tuned by the balanced tuning method versus the desired-model method. Note that some figures are placed in the Supporting Information for better reading due to an enormous number of experiments. 

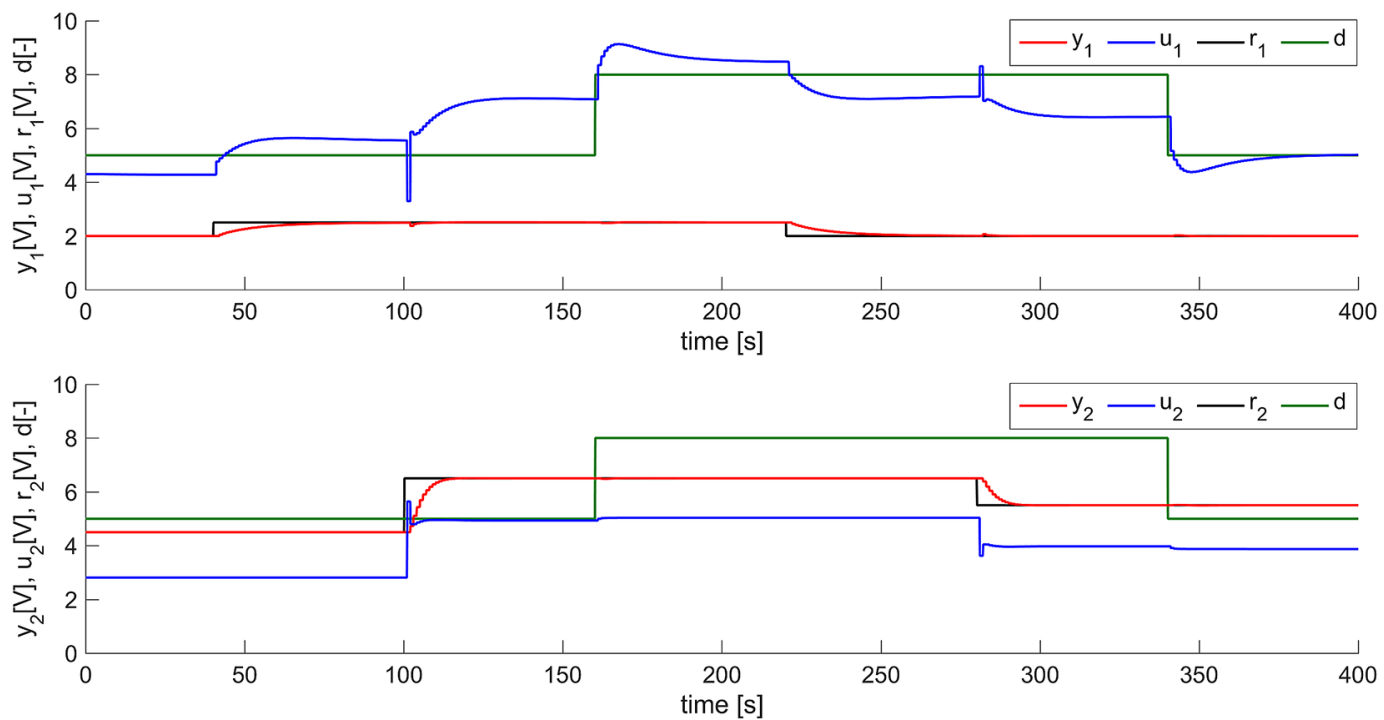

Figure 16. Simulated discrete-time control responses when using decoupling and antidisturbance controllers and $C_{22}(s)$ being set by the balanced tuning method.
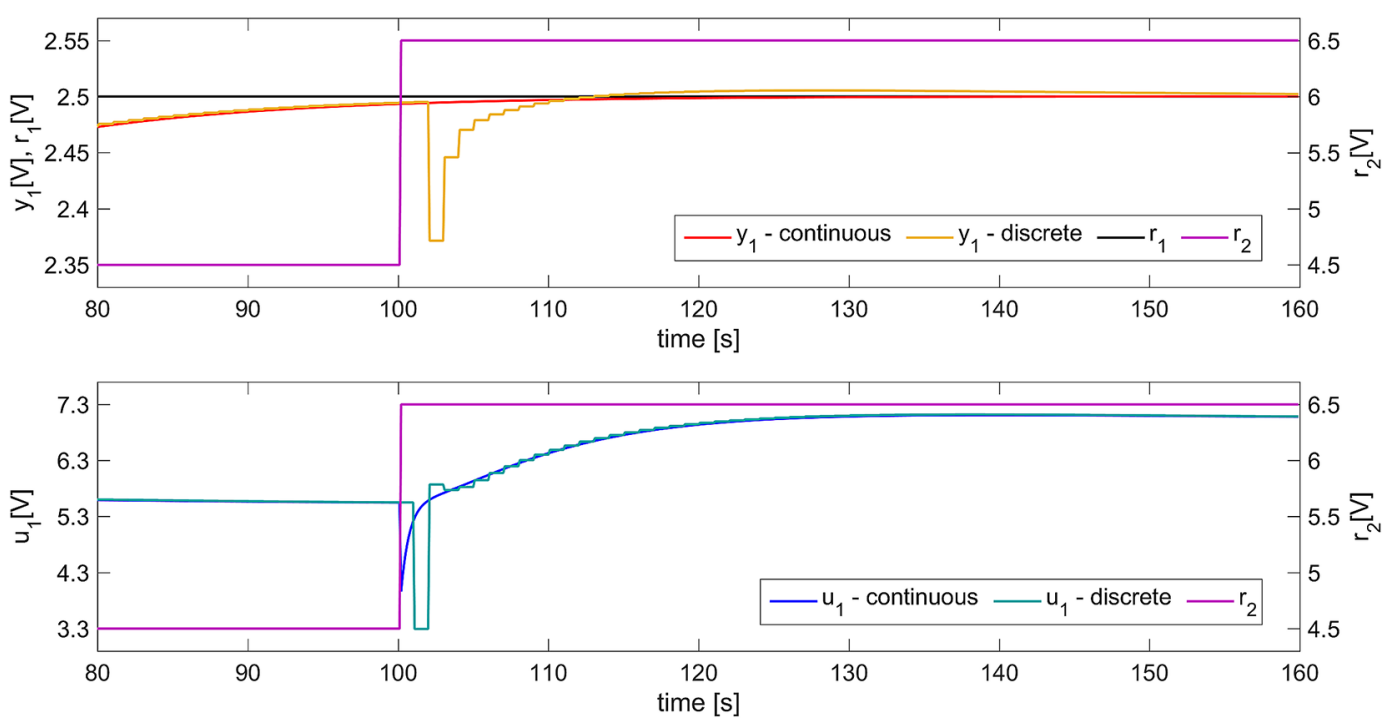

Figure 17. Detailed comparison of Figures 15 and 16-reference tracking.

Although the laboratory model provides voltage outputs, it is desirable for engineers and practitioners to translate these values into temperature and air-flow (i.e., volume per time) ones. Thus, we have calibrated the voltage output from the thermistor via a standard Pt100 temperature sensor and the voltage output from the vane flowmeter using a PCE-THA 10 air flowmeter. The following approximating linear relations have been obtained

$$
\begin{aligned}
& y_{1, \mathrm{~T}}\left[{ }^{\circ} \mathrm{C}\right] \approx 23.6+12.4 y_{1}[\mathrm{~V}] \\
& y_{2, \mathrm{~F}}\left[\mathrm{~m} \mathrm{~s}^{-1}\right] \approx 0.13+0.76 y_{2}[\mathrm{~V}]
\end{aligned}
$$

The reference signal levels for our experiments are $r_{1}=$ $[2.35 ; 2.55] \mathrm{V}$ and $r_{2}=[4.5 ; 6.5] \mathrm{V}$, which approximately implies that $r_{1, \mathrm{~T}}=[52.7 ; 55.3]{ }^{\circ} \mathrm{C}$ and $r_{2, \mathrm{~F}}=[3.55 ; 5.07] \mathrm{m}$ $\mathrm{s}^{-1}$, respectively.

All possible combinations of control system structures and tuning rules yield eight sets of simulated control responses (Figures 15, 16, and S1-S6). Figures 15 and S3 demonstrate by simulation that the simultaneous use of the designed continuous-time primary, decoupling, and antidisturbance controllers satisfies control system stability, almost absolute invariance to disturbances, and ideal rejection of crossinteractions in control loops. However, the undesirable impact of the discretization ( $c 2 d$ Matlab function with $T_{s}=1 \mathrm{~s}$ ) can be seen from a detailed comparison of continuous-time and discrete-time responses (of $u_{1}(t), y_{1}(t)$ ) to step changes of reference and disturbance variables, provided to the reader in Figures 17 and 18.

In more detail, although continuous-time $y_{1}(t)$ remains unaffected by the step change of $r_{2}(t)$ in $t=100 \mathrm{~s}$ due to $\mathbf{G}_{\mathrm{D}}(s)$, there is a small yet abrupt change of discrete-time $y_{1}(t)$ in Figure 17. Similarly, the step change of $d(t)$ in $t=160 \mathrm{~s}$ has only a negligible effect on continuous-time, compared to the discrete-time output (Figure 18).

4.5. Experimental Hot-Air Tunnel Control via PLC. The PLC uses SCADA/HMI for data measurement and archiving. A simple ad hoc SCADA/HMI is developed in the 

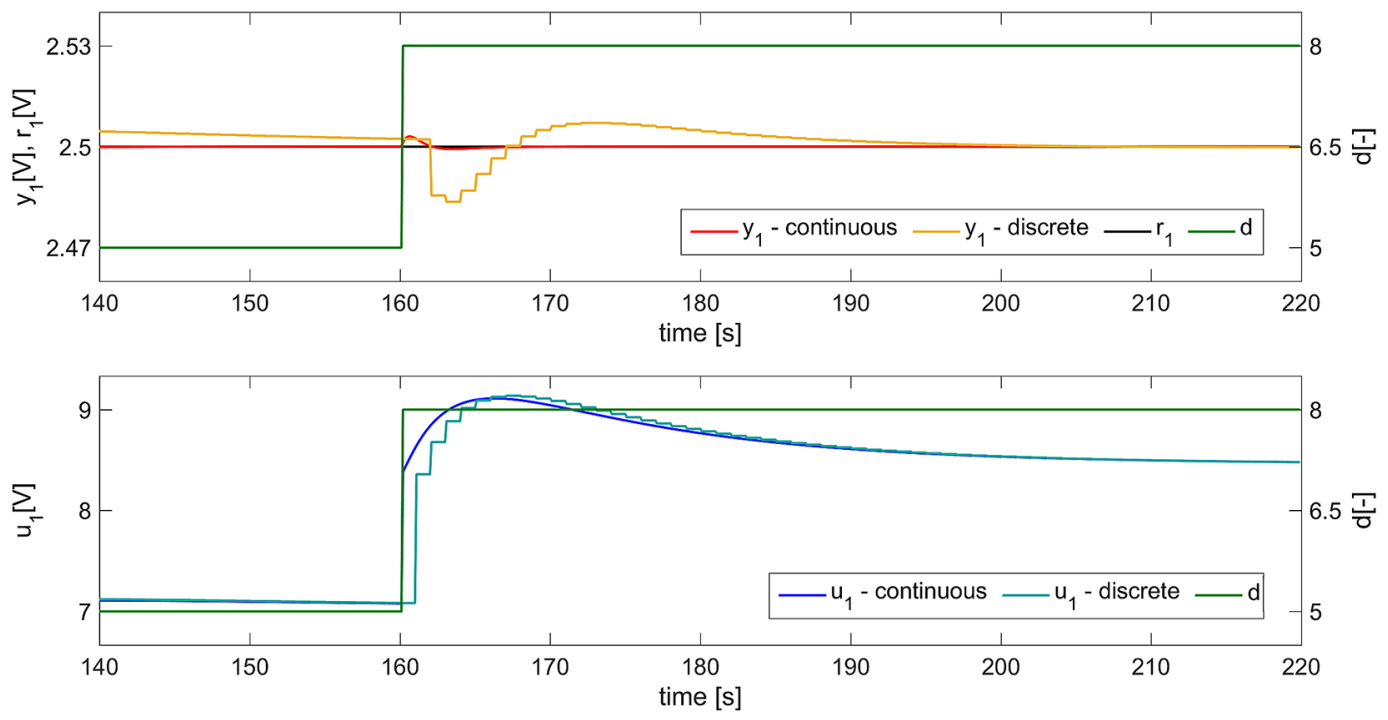

Figure 18. Detailed comparison of Figures 15 and 16-disturbance rejection.

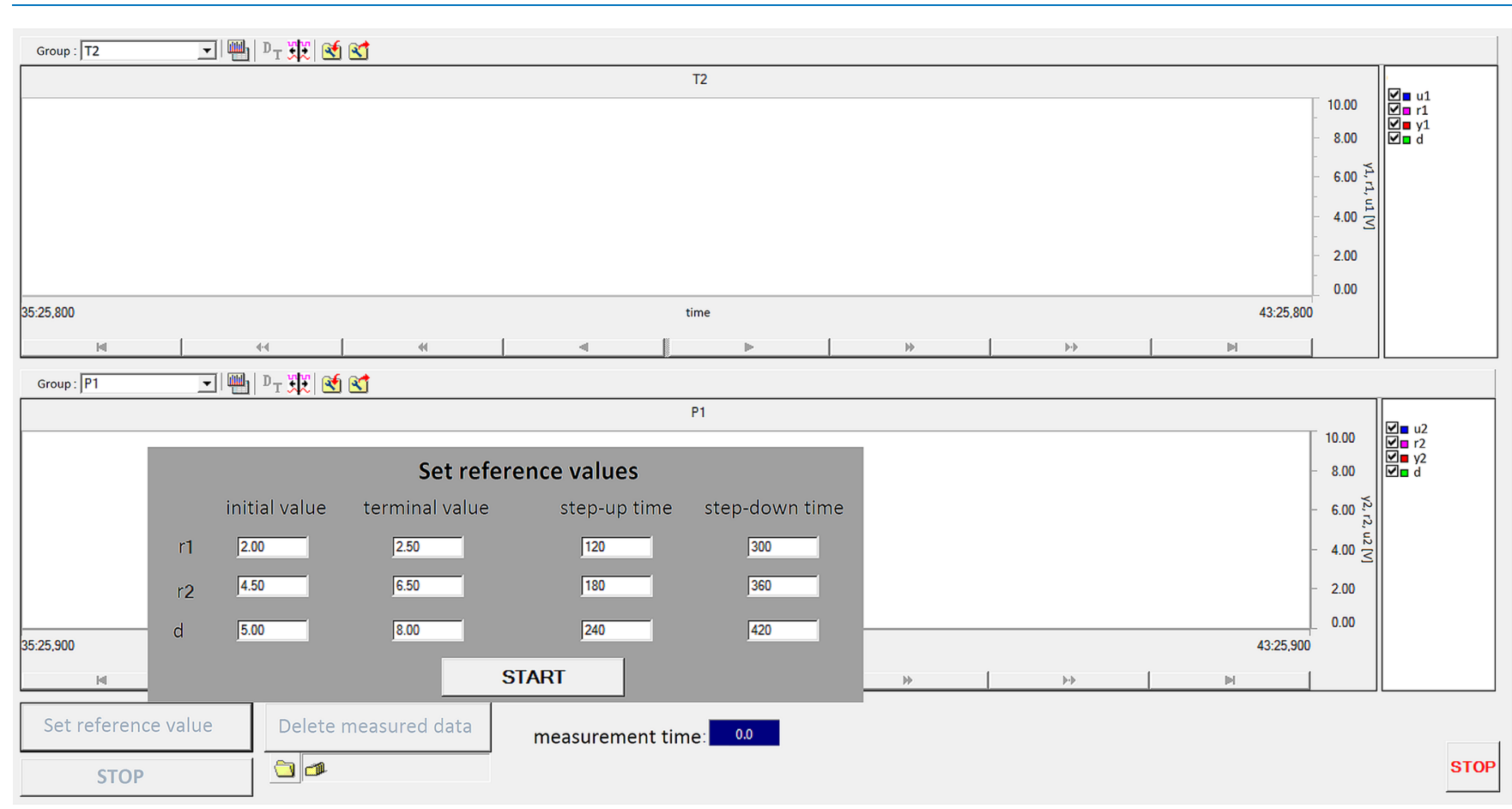

Figure 19. SCADA/HMI developed for the PLC.

Control Web software system ${ }^{76}$ that enables the user to set reference values, display, save or delete the measured data, and start and stop the experiment (see Figure 19).

It is necessary to find controller discrete-time formulations introduced in Section 4.3, where $T_{\mathrm{s}}=1$ is applied. A simple anti-windup calculation based on the reduction of control action difference is used whenever $u_{1}, u_{2}$ are out of bounds $[-10,10] \mathrm{V}$.

Calculated control signals and measured responses are given in Figures 20, 21, S7, and S8. Apparently, all the responses are stable; however, requirements of the ideal cross-interactions decoupling and absolute invariance are met only when both decouplers (39) and (40) and antidisturbance controllers (41) are used. The disturbance rejection satisfaction can be seen from Figure 20 where neither $y_{1}(t)$ nor $y_{2}(t)$ are affected by the step change of $d(t)$ in $t=160 \mathrm{~s}$, due to $\mathbf{G}_{\mathrm{AC}}(s)$. A detailed comparison of responses $u_{1}(t)$ and $y_{1}(t)$ to a step change of $r_{2}(t)$ taken from Figures 20 and 21 are displayed in Figure 22. It is clear from the figure that the step-up change of $r_{2}(t)$ yields a cooling of $y_{1}(t)$. The cooling is more distinct if $\mathbf{G}_{\mathrm{D}}(s)$ is ignored. The initial computed difference in $u_{1}(t)$ is likely caused by a different ambient temperature when controlling.

4.6. Experimental Hot-Air Tunnel Control via $\mathrm{PCI}$ Card and Matlab. The Matlab/Simulink framework is used in combination with the PCI card for real-time measurements as well. It is set $T_{s}=1 \mathrm{~s}$ for $\mathrm{AO}$ and DO; however, the latter uses the pulse width modulation (PWM) with its internal sampling of $10 \mathrm{~ms}$. Therefore, a rate transition block must be used (see Figure 5). Data from AI are measured and stored with the period of $0.1 \mathrm{~s}$ yet $T_{\mathrm{s}}=1 \mathrm{~s}$ again. 

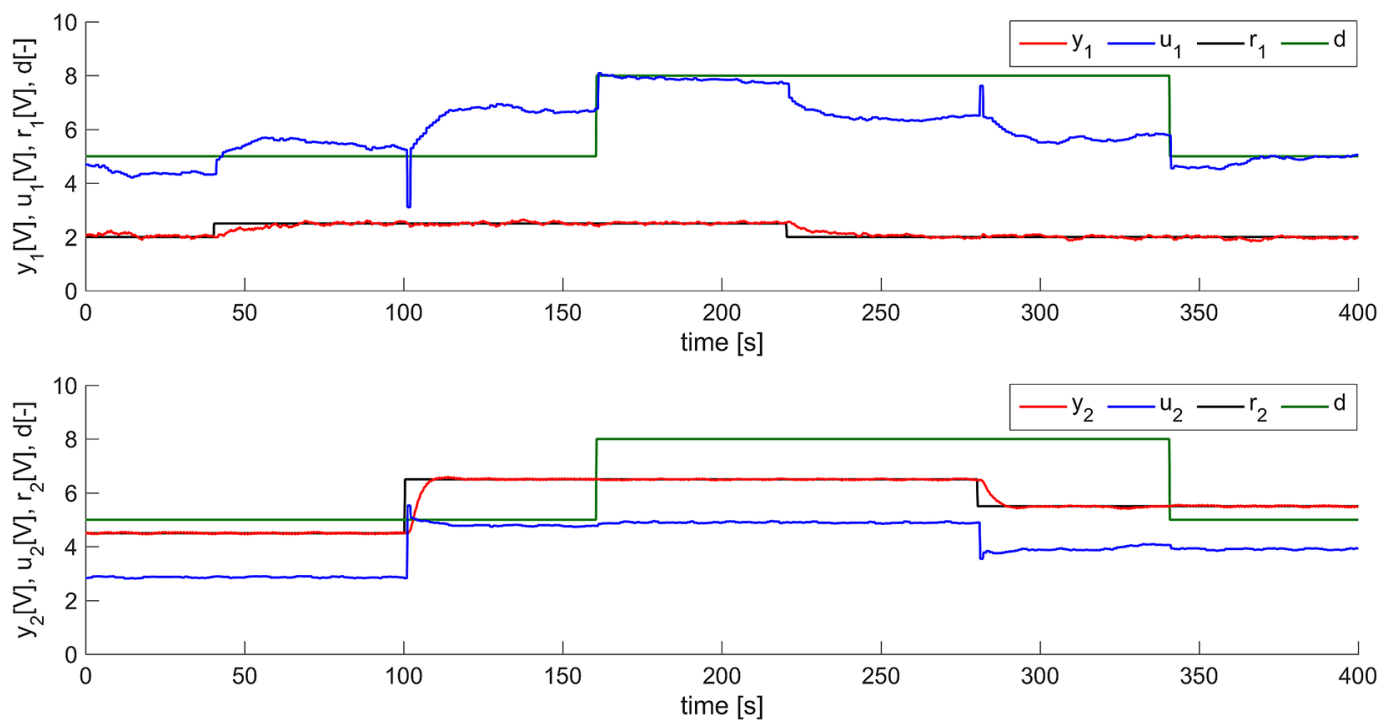

Figure 20. Measured control responses for the PLC when using decoupling and antidisturbance controllers and $C_{22}(s)$ being set by the balanced tuning method.
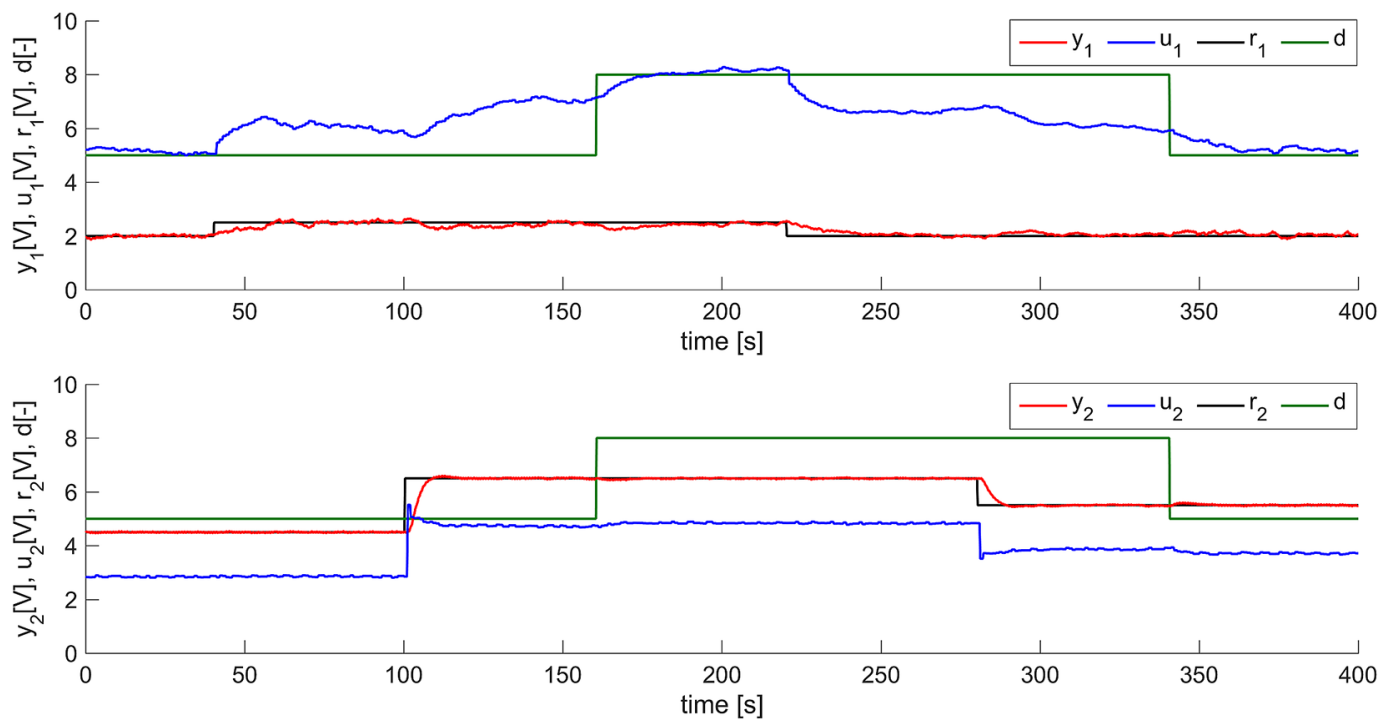

Figure 21. Measured control responses for the PLC without using decoupling or antidisturbance controllers and $C_{22}(s)$ being set by the balanced tuning method.

Controlled plant transfer function matrices are substituted with $\mathrm{AI} / \mathrm{AO}$ to/from the Advantech $\mathrm{PCI}$ card in the discretized Matlab control system scheme (with the noninverted decoupler for simplicity) (see Figure 23). A detailed view under the mask of $\mathbf{G}_{S}(s)$ is shown in Figure 24 .

Corresponding control responses are provided to the reader in Figures 25, 26, S9, and S10. As can be observed, these responses are very close to those obtained by using the PLC.

4.7. Control Response Evaluation. The beneficial use of the designed decoupling and disturbance-rejection elements is compared to the simple scheme that does not include these controllers. The effect of different controller parameter settings is also being observed. In addition, simulated responses are faced with those obtained by the laboratory experiments. Moreover, continuous-time control law formulations are compared to discrete-time ones when simulating. Last but not least, the impact of the use of the PLC versus the PCI card (plus a PC) for practice is evaluated.
4.7.1. Computed Integral Criteria for Evaluation. Integral square error (ISE), integral absolute error (IAE), integral time absolute error (ITAE), and total variation (TV) are computed to evaluate the results. Their continuous-time definitions are given in (54), and the discrete-time definition of $\mathrm{TV}$ is provided in (55).

$$
\begin{aligned}
& J_{\mathrm{ISE}}^{f}:=\int_{t_{0}}^{t_{1}} f^{2}(t) \mathrm{d} t, \quad J_{\mathrm{IAE}}^{f}:=\int_{t_{0}}^{t_{1}}|f(t)| \mathrm{d} t, \\
& J_{\mathrm{ITAE}}^{f}:=\int_{t_{0}}^{t_{1}} t|f(t)| \mathrm{d} t, \quad J_{\mathrm{TV}}^{f}:=\int_{t_{0}}^{t_{1}}|\dot{f}(t)| \mathrm{d} t \\
& J_{\mathrm{TV}, d}^{f}:=\sum_{k=k_{0}+1}^{k_{1}}\left|f\left(t_{k}\right)-f\left(t_{k-1}\right)\right|
\end{aligned}
$$

The criteria are evaluated for the entire experiment, i.e., $t_{0}=$ $k_{0} T_{\mathrm{s}}=40$ and $t_{1}=k_{1} T_{\mathrm{s}}=400 \mathrm{~s}$ for $u_{1}(t)$ and $y_{1}(t)$ and $t_{0}=$ 100 and $t_{1}=400 \mathrm{~s}$ for $u_{2}(t)$ and $y_{2}(t)$. The control performance 

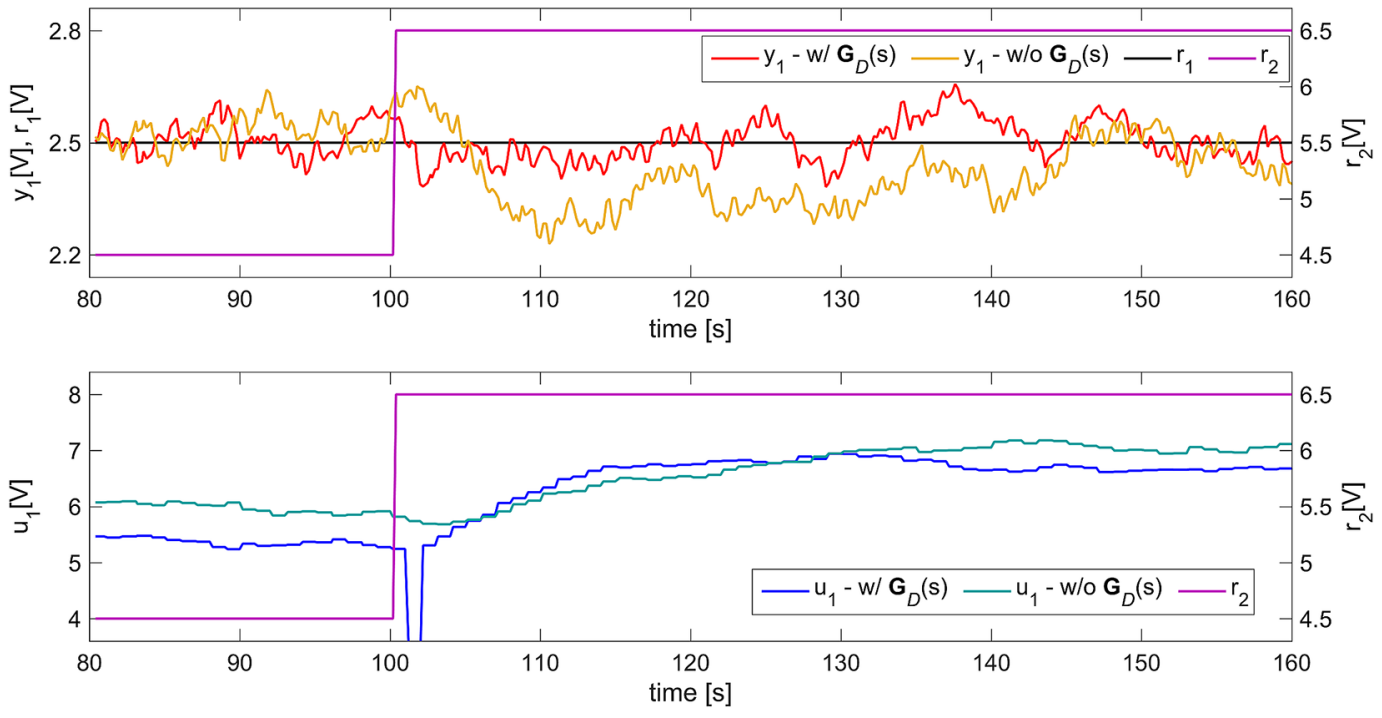

Figure 22. Detailed comparison of Figures 20 and 21-decoupling.

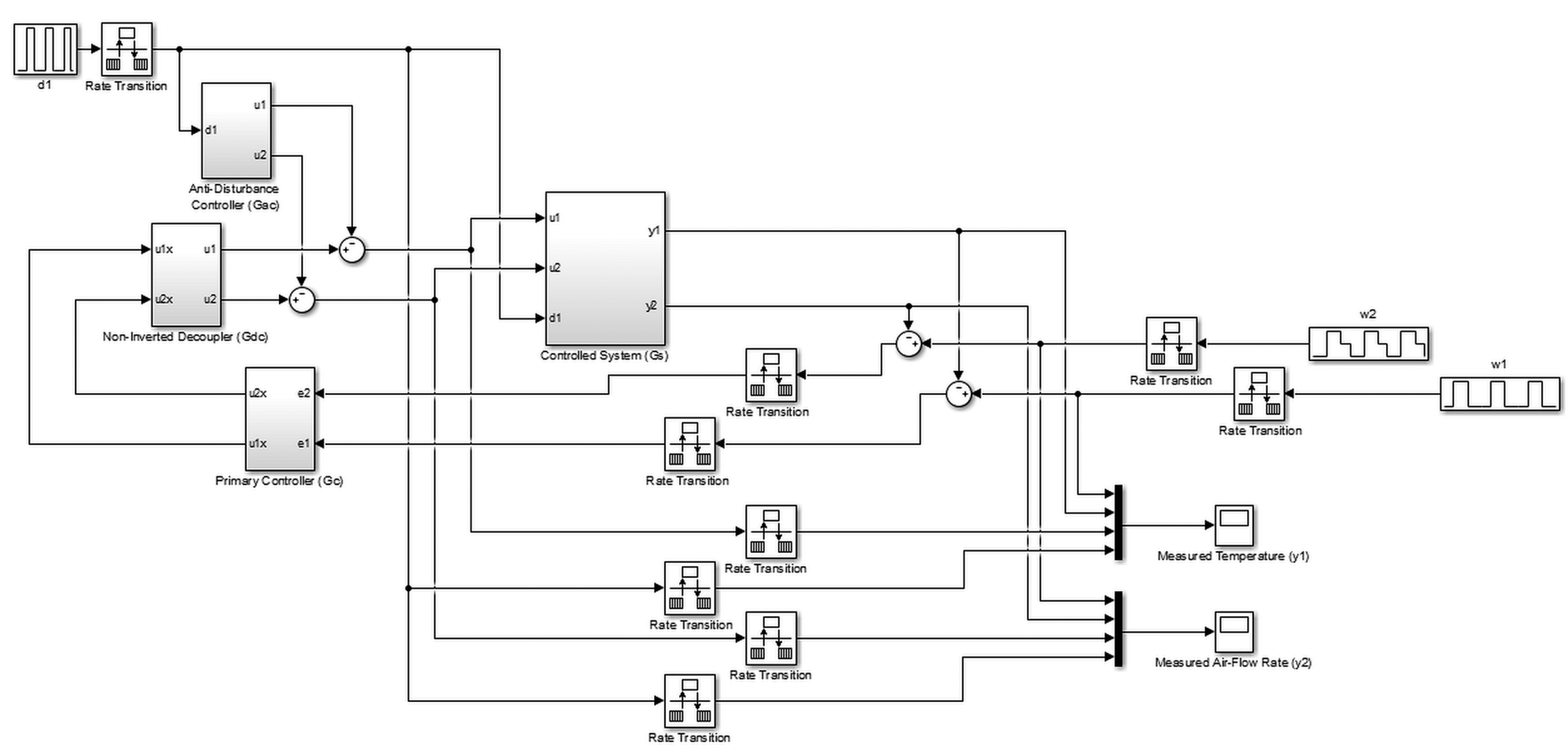

Figure 23. Control system scheme in Matlab/Simulink.

of various responses is computed for $\int_{\mathrm{ISE}}^{e_{i}}, \int_{\mathrm{IAE}}^{e_{i}}, \int_{\mathrm{ITAE}}^{e_{i}}$ and $\int_{\mathrm{ISE}, d}^{e_{i}}$, $J_{\mathrm{IAE}, d}^{e_{i}}, J_{\mathrm{ITAE}, d}^{e_{i}}(i=1,2)$.

The total energy consumption-which is one of the most monitored feature nowadays-is closely related to $J_{\mathrm{ISE}}^{u_{i}}, J_{\mathrm{IAE}}^{u_{i}}$, $J_{\mathrm{ITAE}}^{u_{i}}$ and $J_{\mathrm{ISE}, d}^{u_{i}}, J_{\mathrm{IAE}, d}^{u_{i}}, J_{\mathrm{ITAE}, d}^{u_{i}}(i=1,2)$, while values of $J_{\mathrm{ISE}}^{u_{i}}, J_{\mathrm{IAE}}^{u_{i}}$ $J_{\mathrm{ITAE}}^{i_{i}}, J_{\mathrm{TV}}^{u_{i}}$ and $J_{\mathrm{ISE}, d}^{\Delta u_{i}}, J_{\mathrm{IAE}, d}^{\Delta u_{i}}, J_{\mathrm{ITAE}, d}^{\Delta u_{i}}, J_{\mathrm{TV}, d}^{u_{i}}(i=1,2)$ correspond to expected lifetime. Notice that $J_{\mathrm{IAE}}^{\dot{u}}$ and $J_{\mathrm{TV}}^{u}$ coincide in the continuous-time case. In the discrete-time case, it does not exactly hold that $J_{\mathrm{IAE}, d}^{\Delta u}=J_{\mathrm{TV}, d}^{u} T_{s}$ in our experiments since $\int_{\mathrm{IAE}, d}^{f}$ (and also other integral criteria) are computed with the Matlab integration block, which uses the trapezoidal quadrature rule, while $f_{\mathrm{TV}, d}^{f}$ agrees with the most straightforward rectangle formula.

Table 1 assigns Roman numerals to particular feedback control systems and simulated and real-life experiments for better readability of other tables with criteria values. Computed values of $\int_{\mathrm{ISE}}^{e_{i}}, J_{\mathrm{IAE}}^{e_{i}}, J_{\mathrm{ITAE}}^{e_{i}}$ and $J_{\mathrm{ISE}, d}^{e_{i}}, J_{\mathrm{IAE}, d}^{e_{i}}, J_{\mathrm{ITAE}, d}^{e_{i}}(i$
$=1,2$ ) obtained using the balanced tuning and desired-model methods for setting $C_{22}(s)$ are summarized in Tables 2 and 3, respectively. The values of $J_{\mathrm{ISE}}^{u_{i}}, J_{\mathrm{IAE}}^{u_{i}}, J_{\mathrm{ITAE}}^{u_{i}}\left(J_{\mathrm{ISE}, d}^{u_{i}}, J_{\mathrm{IAE}, d}^{u_{i}}, J_{\mathrm{ITAE}, d}^{u_{i}}\right)$ and $J_{\mathrm{ISE}}^{u_{i}}, J_{\mathrm{IAE}}^{u_{i}}, J_{\mathrm{ITAE}}^{\dot{u}_{i}}=J_{\mathrm{TV}}^{u_{i}}\left(J_{\mathrm{ISE}, d}^{\Delta u_{i}}, J_{\mathrm{IAE}, d}^{\Delta u_{i}}, J_{\mathrm{ITAE}, d}^{\Delta u_{i}}, J_{\mathrm{TV}, d}^{u_{i}}\right)$ for the two tuning rules are given to the reader in Tables 4-7, respectively.

4.7.2. Criteria Value Analysis. Let us analyze the data presented in Tables 2-34567 regarding the control performance and effort and energy efficiency.

It can be deduced from Tables 2 and 3 that the simulated continuous-time control system with decouplers and antidisturbance controllers (scenario I) gives the best performance criteria values for temperature control. However, the performance improvement for $e_{2}(t)$ when using decoupling and antidisturbance controllers is less evident or even disputable. Simulated discrete-time responses (scenario II) yield superior performance by $24.3 \%$ on average compared to the use of the 


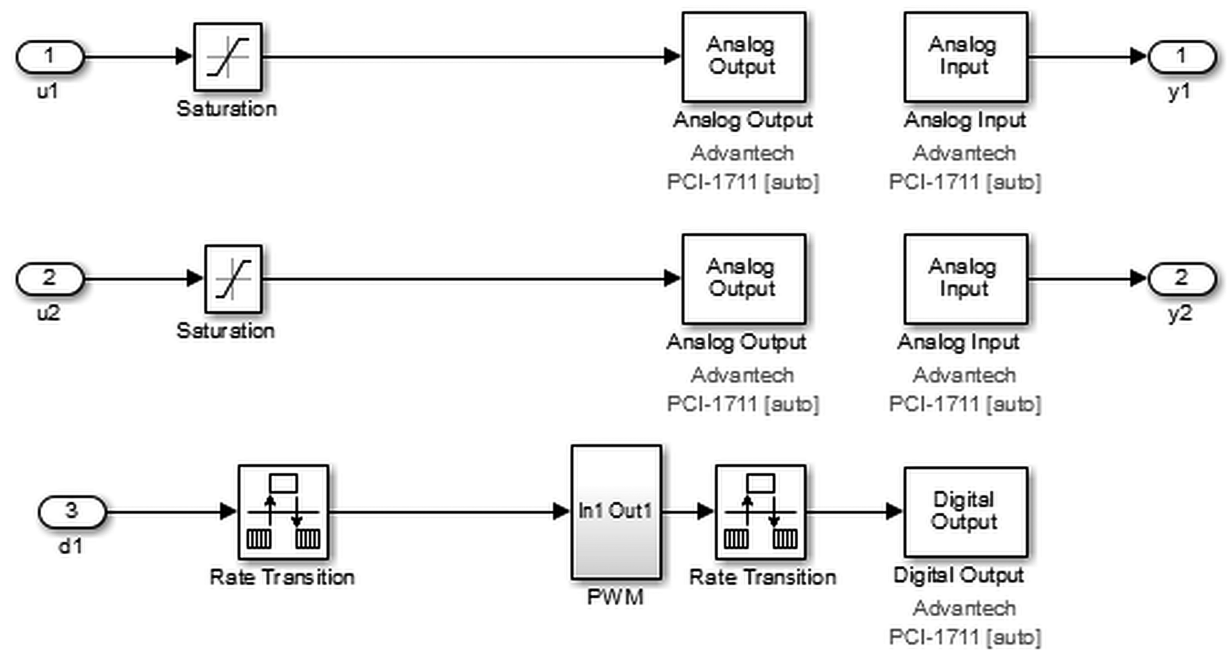

Figure 24. Detailed view under the mask of $\mathbf{G}_{S}(s)$.
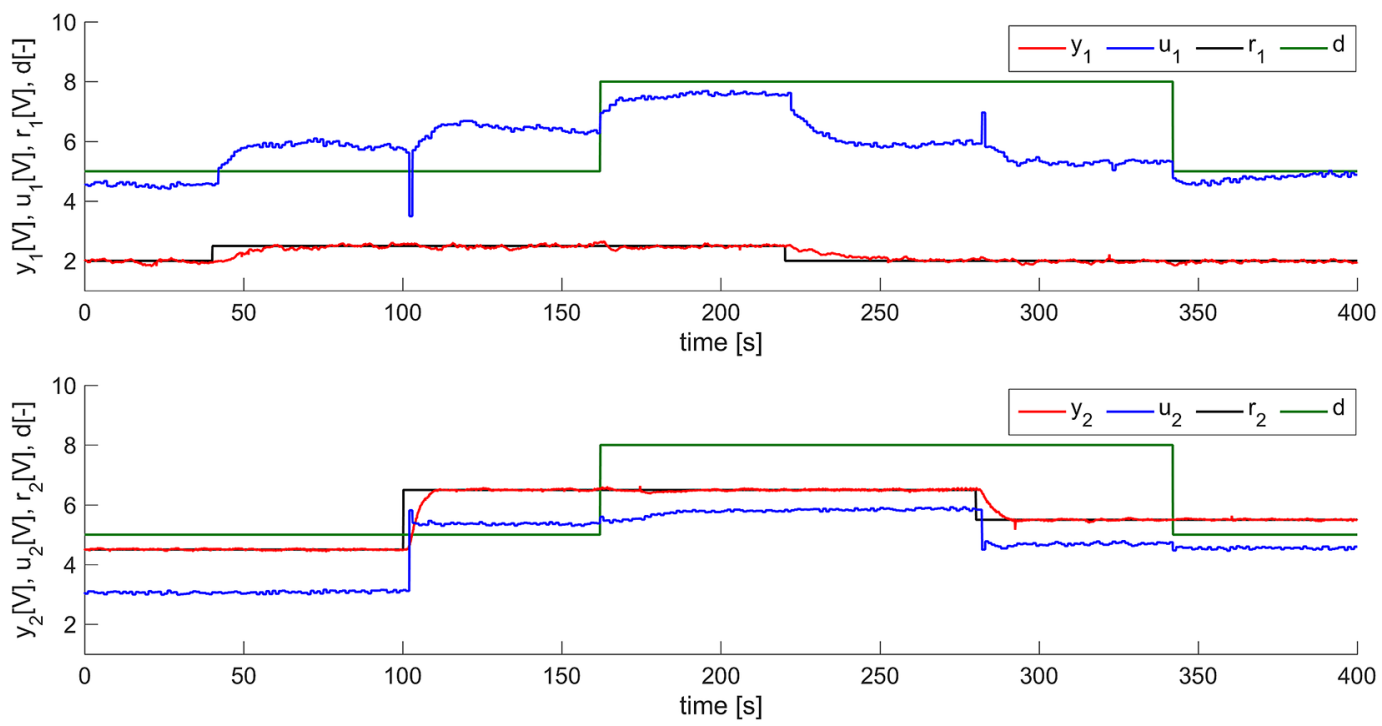

Figure 25. Measured control responses for the PCI card when using decoupling and antidisturbance controllers and $C_{22}(s)$ being set by the balanced tuning method.

PLC or the PCI card in real-life experiments (scenarios III and IV) since they are not affected by the external fluctuations, measurement noise, data processing, etc.

Contrariwise, when neither decouplers nor antidisturbance controllers are used for laboratory experiments (scenarios VII and VIII), criteria values for $e_{1}(t)$ are lower (by $12.8 \%$ on average) than those of simulated responses as the impact of $d(t)$ on temperature is scanty. Although the benefit of both the additional controllers in laboratory experiments (scenarios III and IV) for $e_{1}(t)$ is substantial (by $31.3 \%$ on average and even by $35.3 \%$ when using the balanced tuning), their effect on flow rate control is disputable. Regarding the main subject of study-i.e., the experimental verification of the proposed control system design-the use of PLC gives clearly better performance measures for both temperature and air-flow control.

From ISE and IAE criteria values, it cannot be unambiguously deduced which of the benchmarked controller tuning rules applied to $C_{22}(s)$ provides better results. However, the ITAE criterion proves most significantly that using the balanced tuning method is more appropriate for control of the hot-air tunnel from the performance point of view. The average advantage of the balanced tuning method is only $4.4 \%$ for simulation and $7.6 \%$ for experimental results throughout all the performance criteria.

Data in Tables 4 and 5 closely related to energy efficiency that represents a significant touchstone nowadays. Real-life experiments imply that the overall energy consumption when using control systems with decouplers and antidisturbance controllers (scenarios III and IV) is less than that in the opposite case. The average energy-consumption improvement renders $3.8 \%$ when using the balanced tuning and $1.9 \%$ for the desired-model method. Although this outcome does not hold true for $u_{2}(t)$ in some criteria, the measured data for $u_{1}(t)$ (i.e., the heating bulb input voltage) are abundantly clear; especially when the primary controllers are tuned by the balanced method. When taking into account that $u_{1}(t)$ is more energy demanding, the absolute energy consumption decreases by $6.0 \%$ and $2.7 \%$, respectively. Besides, the use of the PLC yields better results again. Surprisingly, simulated response results are opposed to the measured ones; however, 

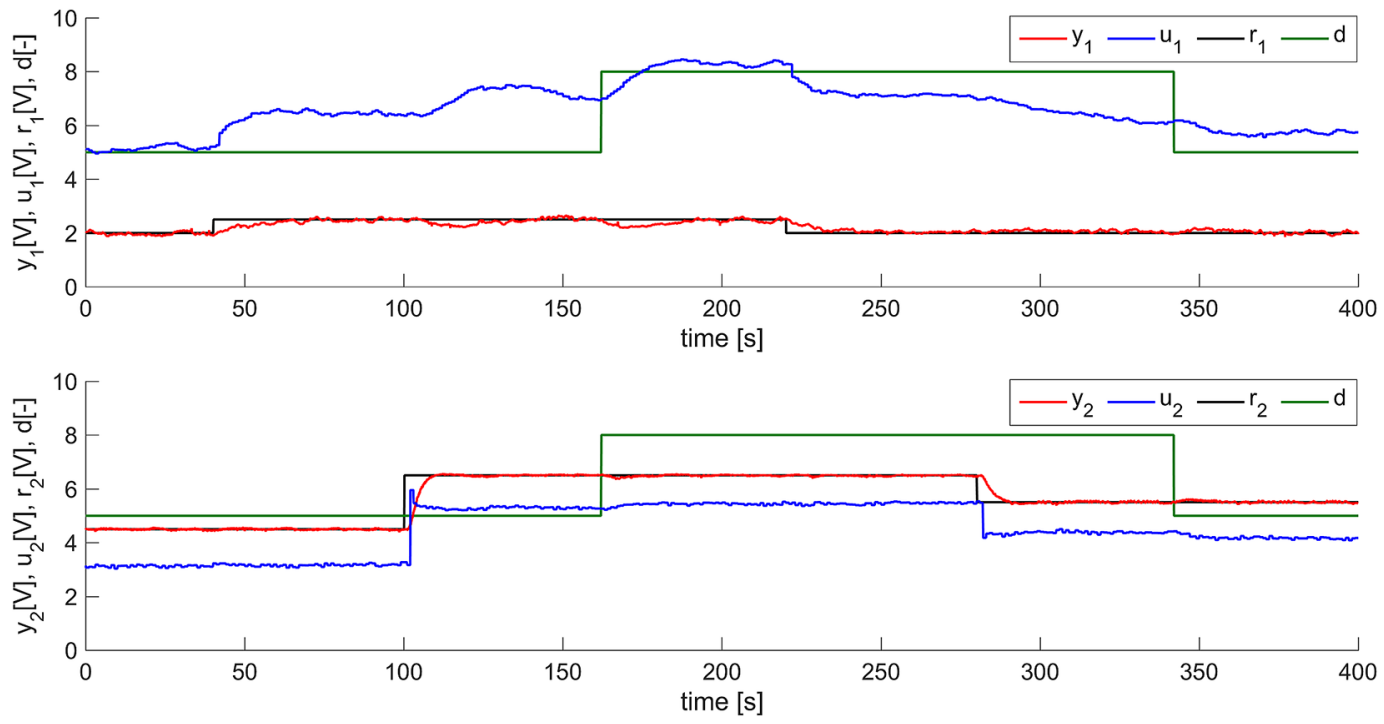

Figure 26. Measured control responses for the PCI card without using decoupling or antidisturbance controllers and $C_{22}(s)$ being set by the balanced tuning method.

Table 1. Numbers Assigned to Control Systems, Simulations, and Real-Life Measurements

\begin{tabular}{ll} 
control system and simulation/experiment & assigned number \\
with $\mathbf{G}_{\mathrm{D}}(s)$ and $\mathbf{G}_{\mathrm{AC}}(s)$ & \\
simulated continuous-time control & $\mathrm{I}$ \\
simulated discrete-time control & $\mathrm{II}$ \\
measured control via PLC & III \\
measured control via PCI card & IV \\
without $\mathbf{G}_{\mathrm{D}}(s)$ or $\mathbf{G}_{\mathrm{AC}}(s)$ & \\
simulated continuous-time control & $\mathrm{V}$ \\
simulated discrete-time control & VI \\
measured control via PLC & VII \\
measured control via PCI card & VIII \\
\hline
\end{tabular}

Table 2. Values of $J_{\text {ISE }}^{e_{i}}, J_{\text {IAE }}^{e_{i}}, J_{\text {ITAE }}^{e_{i}}\left(J_{\text {ISE }, d}^{e_{i}}, J_{\text {IAE, }, d}^{e_{i}}, J_{\text {ITAE }, d}^{e_{i}}\right)$ for $C_{22}(s)$ Set by the Balanced Tuning Method

\begin{tabular}{lccrccc} 
expt no. & $\int_{\text {ISE }}^{e_{1}}$ & $\int_{\text {IAE }}^{e_{1}}$ & \multicolumn{1}{c}{$\int_{\text {ITAE }}^{e_{1}}$} & $\int_{\text {ISE }}^{e_{2}}$ & $J_{\text {IAE }}^{e_{2}}$ & $J_{\text {ITAE }}^{e_{2}}$ \\
I & 2.87 & 12.64 & 0.80 & 13.71 & 13.93 & 35.22 \\
II & 3.71 & 14.87 & 6.39 & 19.06 & 16.87 & 41.00 \\
III & 4.30 & 25.23 & 62.13 & 15.73 & 19.44 & 40.18 \\
IV & 5.61 & 28.09 & 58.61 & 17.03 & 22.37 & 41.23 \\
V & 7.57 & 38.86 & 164.40 & 13.32 & 14.22 & 35.22 \\
VI & 8.51 & 40.28 & 167.56 & 19.24 & 17.33 & 41.00 \\
VII & 6.82 & 38.53 & 135.78 & 14.98 & 18.35 & 39.01 \\
VIII & 6.45 & 35.92 & 119.86 & 15.98 & 21.60 & 43.34
\end{tabular}

Table 3. Values of $J_{\text {ISE }}^{e_{i}}, J_{\text {IAE }}^{e_{i}}, J_{\text {ITAE }}^{e_{i}}\left(J_{\text {ISE, }, d}^{e_{i}}, J_{\text {IAE, }, d}^{e_{i}}, J_{\text {ITAE }, d}^{e_{i}}\right)$ for $\mathrm{C}_{22}(\mathrm{~s})$ Set by the Desired-Model Method

\begin{tabular}{lccrccc} 
expt no. & $\int_{\text {ISE }}^{e_{1}}$ & $\int_{\text {IAE }}^{e_{1}}$ & \multicolumn{1}{c}{$\int_{\text {ITAE }}^{e_{1}}$} & $\int_{\text {ISE }}^{e_{2}}$ & \multicolumn{1}{c}{$\int_{\text {IAE }}^{e_{2}}$} & $J_{\text {ITAE }}^{e_{2}}$ \\
I & 2.89 & 12.78 & 0.91 & 12.56 & 15.09 & 51.10 \\
II & 3.58 & 14.24 & 5.89 & 18.56 & 18.40 & 62.01 \\
III & 4.68 & 28.03 & 111.16 & 14.23 & 17.73 & 40.79 \\
IV & 4.50 & 27.61 & 78.36 & 17.44 & 25.24 & 64.12 \\
V & 7.50 & 38.88 & 109.56 & 12.47 & 15.80 & 51.59 \\
VI & 8.50 & 38.53 & 137.71 & 18.15 & 18.35 & 67.29 \\
VII & 6.13 & 32.40 & 163.16 & 13.84 & 19.42 & 51.10 \\
VIII & 6.32 & 31.97 & 166.44 & 17.68 & 24.19 & 62.01
\end{tabular}

Table 4. Values of $J_{\mathrm{ISE}}^{u_{i}}, J_{\mathrm{IAE}}^{u_{i}}, J_{\mathrm{ITAE}}^{u_{i}}\left(J_{\mathrm{ISE}, d}^{u_{i}}, J_{\mathrm{IAE}, d}^{u_{i}}, J_{\mathrm{ITAE}, d}^{u_{i}}\right)$ for $C_{22}(s)$ Set by the Balanced Tuning Method

\begin{tabular}{|c|c|c|c|c|c|c|}
\hline $\begin{array}{c}\text { expt } \\
\text { no. }\end{array}$ & $\left(\cdot \int_{\text {ISE }}^{u_{1}}\right)$ & $\left(\cdot 10^{3}\right)$ & $\left(\cdot 10^{3}\right)$ & $\left(\cdot 10^{3}\right)$ & $\left(\cdot \int_{\mathrm{IAE}}^{u_{2}}\right)$ & $\left(\cdot 10^{3}\right)$ \\
\hline I & 17.00 & 2.548 & 8.759 & 7.143 & 1.653 & 6.173 \\
\hline II & 17.04 & 2.551 & 8.771 & 7.140 & 1.652 & 6.173 \\
\hline III & 14.81 & 2.391 & 8.378 & 6.910 & 1.630 & 5.993 \\
\hline IV & 13.97 & 2.334 & 8.074 & 9.241 & 1.876 & 6.702 \\
\hline V & 16.79 & 2.541 & 8.347 & 7.142 & 1.653 & 6.173 \\
\hline VI & 16.77 & 2.540 & 8.335 & 7.141 & 1.652 & 6.173 \\
\hline VII & 16.57 & 2.548 & 8.599 & 6.697 & 1.605 & 5.929 \\
\hline VIII & 18.13 & 2.669 & 9.094 & 8.427 & 1.799 & 6.619 \\
\hline
\end{tabular}

Table 5. Values of $J_{\text {ISE }}^{u_{i}}, J_{\text {IAE }}^{u_{i}}, J_{\text {ITAE }}^{u_{i}}\left(J_{\text {ISE, }, d}^{u_{i}}, J_{\text {IAE, }, d}^{u_{i}}, J_{\text {ITAE }, d}^{u_{i}}\right)$ for $C_{22}(s)$ Set by the Desired-Model Method

\begin{tabular}{|c|c|c|c|c|c|c|}
\hline $\begin{array}{l}\text { expt } \\
\text { no. }\end{array}$ & $\begin{array}{l}\left.J_{\mathrm{ISE}}^{u_{1}} \cdot 10^{3}\right) \\
\text { (2) }\end{array}$ & $\left(\cdot 10^{u_{\mathrm{AAE}}}\right)$ & $\begin{array}{l}J_{\mathrm{ITAE}}^{u_{1}} \\
\left(\cdot 10^{3}\right)\end{array}$ & $\left(\cdot 10^{3}\right)$ & $\begin{array}{l}\left.J_{\mathrm{IAE}}^{u_{2}} \cdot 10^{3}\right) \\
\text { (2) }\end{array}$ & $\begin{array}{l}J_{\mathrm{ITAE}}^{u_{2}}\left(\cdot 10^{3}\right) \\
(-1)\end{array}$ \\
\hline I & 16.97 & 2.544 & 8.737 & 7.130 & 1.651 & 6.155 \\
\hline II & 17.05 & 2.552 & 8.746 & 7.094 & 1.647 & 6.154 \\
\hline III & 19.34 & 2.748 & 9.202 & 6.839 & 1.619 & 6.037 \\
\hline IV & 20.54 & 2.844 & 9.848 & 8.711 & 1.832 & 6.689 \\
\hline V & 16.79 & 2.541 & 8.324 & 7.127 & 1.651 & 6.155 \\
\hline VI & 16.77 & 2.541 & 8.312 & 7.095 & 1.647 & 6.154 \\
\hline VII & 20.96 & 2.876 & 9.414 & 6.984 & 1.637 & 6.098 \\
\hline VIII & 21.10 & 2.890 & 9.927 & 8.641 & 1.821 & 6.687 \\
\hline
\end{tabular}

the control impact on practice represents a much more meaningful target.

Control effort expressing the rate of actuators reconfiguration is given in Tables 6 and 7. It can be generally deduced that the better the energy efficiency is, the higher is the control effort required. Indeed, the proposed control design yields higher effort than the simple scheme without decouplers and antidisturbance controllers by nearly $24 \%$ for both the manipulated inputs. In mechanical systems, the higher control effort usually implies the lower lifetime of actuators. The primary fan voltage input is considered as $u_{2}(t)$ for the hot-air tunnel, which corresponds to the rotation speed representing the mechanical stress. Thus, the assessment of the control effort of $u_{2}(t)$ is substantial. Gratifyingly, the 
Table 6. Values of $J_{\text {ISE }}^{i_{i}}, J_{\mathrm{IAE}}^{i_{i}}, J_{\mathrm{ITAE}}^{\dot{u}_{i}}=J_{\mathrm{TV}}^{u_{i}}\left(J_{\mathrm{ISE}, d}^{\Delta u_{i}} J_{\mathrm{IAE}, d}^{\Delta u_{i}}, J_{\mathrm{ITAE}, d}^{\Delta u_{i}}, J_{\mathrm{TV}, d}^{u_{i}}\right)$ for $C_{22}(s)$ Set by the Balanced Tuning Method

\begin{tabular}{lcccccccrr} 
expt no. & $J_{\text {ISE }}^{i_{1}}$ & $J_{\text {IAE }}^{i_{1}}$ & $J_{\text {ITAE }}^{i_{1}}$ & $J_{\text {TV }}^{u_{1}}$ & $J_{\text {ISE }}^{i_{2}}$ & $J_{\text {IAE }}^{i_{2}}$ & $J_{\text {ITAE }}^{u_{2}}$ & \multicolumn{1}{c}{$J_{\text {TV }}^{u_{2}}$} \\
I & 0.502 & 2.325 & 3.522 & 2.325 & 0.698 & 0.695 & 0.480 & 0.695 \\
II & 2.367 & 3.333 & 3.375 & 3.333 & 1.176 & 1.179 & 0.760 & 1.179 \\
III & 1.979 & 4.397 & 12.29 & 23.749 & 1.268 & 1.754 & 5.988 & 10.050 \\
IV & 1.855 & 6.144 & 14.71 & 32.814 & 2.228 & 3.766 & 9.648 & 20.247 \\
V & 0.053 & 1.542 & 6.114 & 1.542 & 0.702 & 0.695 & 0.480 & 0.695 \\
VI & 0.119 & 1.588 & 5.999 & 1.588 & 1.174 & 1.176 & 0.760 & 1.176 \\
VII & 0.161 & 2.987 & 9.220 & 16.088 & 1.431 & 2.597 & 6.460 & 13.640 \\
VIII & 0.155 & 2.926 & 10.09 & 16.016 & 2.138 & 3.767 & 10.24 & 20.364
\end{tabular}

Table 7. Values of $J_{\mathrm{ISE}}^{i_{i}}, J_{\mathrm{IAE}}^{i_{i}}, J_{\mathrm{ITAE}}^{i_{i}}=J_{\mathrm{TV}}^{u_{i}}\left(J_{\mathrm{ISE}, d}^{\Delta u_{i}}, J_{\mathrm{IAE}, d}^{\Delta u_{i}}, J_{\mathrm{ITAE}, d}^{\Delta u_{i}} J_{\mathrm{TV}, d}^{u_{i}}\right)$ for $C_{22}(s)$ Set by the Desired-Model Method

$\begin{array}{lcccccccc}\text { expt no. } & J_{\text {ISE }}^{u_{1}} & J_{\text {IAE }}^{i_{1}} & J_{\text {ITAE }}^{u_{1}} & J_{\text {TV }}^{u_{1}} & J_{\text {ISE }}^{i_{2}} & J_{\text {IAE }}^{i_{2}} & J_{\text {ITAE }}^{u_{2}} & J_{\text {TV }}^{u_{2}} \\ \text { I } & 1.102 & 2.721 & 3.577 & 2.721 & 1.955 & 1.645 & 1.027 & 1.645 \\ \text { II } & 5.060 & 4.207 & 3.412 & 4.207 & 1.181 & 1.186 & 1.897 & 7.504 \\ \text { III } & 4.625 & 3.845 & 7.826 & 34.359 & 3.283 & 3.134 & 21.196 \\ \text { IV } & 3.407 & 7.880 & 19.05 & 42.854 & 2.228 & 5.564 & 12.98 & 30.643 \\ \text { V } & 0.052 & 1.560 & 6.142 & 1.560 & 1.583 & 1.361 & 1.027 & 1.361 \\ \text { VI } & 0.112 & 1.609 & 6.052 & 1.609 & 1.180 & 1.183 & 1.898 & 1.183 \\ \text { VII } & 0.182 & 2.936 & 10.04 & 17.059 & 3.296 & 3.110 & 5.342 & 16.377 \\ \text { VIII } & 0.159 & 2.649 & 10.24 & 14.491 & 2.138 & 5.547 & 14.62 & 29.916\end{array}$

property in question for the proposed control system design is $17.1 \%$ (measured by IAE, ISE, and ITAE) and 13.5\% (measured by TV) less when using the PLC (scenario III) with tuning the primary controller by the balanced method (which represents the best above-evaluated combination of the controller hardware and the tuning rule). Indeed, this tuning method not only should minimize the overshoot but also ensure "a balance" between the proportional and the the integral action and save actuators. ${ }^{67}$ Other combinations give worse data.

On the contrary, the heating bulb input voltage $u_{1}(t)$ requires higher control effort (but better efficiency); yet the actuator is robust to control action changes due to the absence of mechanical parts.

\section{CONCLUSIONS}

This paper was aimed at the multivariable control design and experimental investigation for a hot-air tunnel that is widely used in the industry and technological processes. The proposed decentralized control design incorporates ideal decouplers of cross-interactions that are formulated in inverted and noninverted versions. Explicit relations between these two versions and the standard inverted decoupling scheme were provided. A simple open-loop absolute rejection of measurable disturbances was designed as well. The considered hot-air tunnel includes two manipulated inputs (a heating bulb input voltage and the primary fan input voltage), two controlled outputs (a measuring thermistor voltage and a vane flowmeter), and one disturbance input (secondary fan dimensionless input value). Static and dynamic responses were measured to get a linear operating range and select a suitable linear model, respectively. Consequently, the simple LMS identification procedure and the standard Matlab identification toolbox were used to estimate model parameters. These experiments were done by using a PLC and a PCI card plus a PC. Based on the eventual TITO model, primary, decoupling, and antidisturbance controllers were set. The primary ones were tuned by applying an algebraic polynomial approach, the balanced tuning and desired-model methods.
Discrete-time formulations of controllers and the controlled plant model were proposed to incorporate the used digital hardware tools. Feasible digital implementation could be obtained even if the continuous-time antidisturbance controllers had nonfeasible forms. Finally, simulation and real-life experiments using the PLC and the PCI card were made, and the efficiency, performance, and control effort of several scenarios were evaluated via some integral criteria. The following were especially observed:

(1) The investigated particular control problem showed that the use of a PLC provided a better performance than using a PC equipped with the PCI card in practice.

(2) Laboratory experiments with both the PLC and a PC equipped with the PCI card and Matlab can be sufficiently used to control the air-heat tunnel, large-scale versions of which are widely used in the industry.

(3) By benchmark of using two tuning rules, the balanced tuning method yields superior control responses compared to the desired-model setting in this study

(4) The simultaneous use of the designed decouplers and antidisturbance controllers provides significantly better results compared to the simple coupled feedback control system in practice. Temperature control performance measured by integral criteria is increased by $35.3 \%$, energy consumption and control effort of mechanical actuator parts are lower by up to $6 \%$ and by $17.1 \%$, respectively (when using the PLC and the balanced tuning). A relatively high control effort for the heating bulb represents the only nonpositive feature.

The intention of the work was not to determine suitable or optimal temperature or air-flow setpoints that depends on a particular process or technology.

Although the effect of delays and latencies in the model has not been investigated in this study, the closed-loop effect of delays can be undesirable in thermal control systems. A possible solution in these cases can be the use of algebraic model-based control design approaches based on a special ring of quasi-polynomial meromorphic functions. ${ }^{77}$ Hence, the consideration of plant delays and latencies in the control 
design or the attenuation of unmeasurable disturbances might pose possible future research directions.

\section{ASSOCIATED CONTENT}

\section{SI Supporting Information}

The Supporting Information is available free of charge at https://pubs.acs.org/doi/10.1021/acsomega.1c02239.

Simulated continuous-time control responses and simulated discrete-time control responses without using decoupling or antidisturbance controllers and $C_{22}(s)$ being set by the balanced tuning method; simulated continuous-time control responses and simulated discrete-time control responses when using decoupling and antidisturbance controllers and $C_{22}(s)$ being set by the desired-model method; simulated continuous-time control responses and simulated discrete-time control responses without using decoupling or antidisturbance controllers and $C_{22}(s)$ being set by the desired-model method; measured control responses for the PLC when using decoupling and antidisturbance controllers and $C_{22}(s)$ being set by the desired-model method; measured control responses for the PLC without using decoupling or antidisturbance controllers and $C_{22}(s)$ being set by the desired-model method; measured control responses for the PCI card when using decoupling and antidisturbance controllers and $C_{22}(s)$ being set by the desired-model method; and measured control responses for the PCI card without using decoupling or antidisturbance controllers and $C_{22}(s)$ being set by the desired-model method (PDF)

\section{AUTHOR INFORMATION}

\section{Corresponding Author}

Libor Pekař - Department of Automation and Control Engineering, Faculty of Applied Informatics, Tomas Bata University in Zlín, 76005 Zlín, Czech Republic;

Email: pekar@utb.cz

\section{Authors}

Pavel Navrátil - Department of Automation and Control Engineering, Faculty of Applied Informatics, Tomas Bata University in Zlín, 76005 Zlín, Czech Republic

Radek Matušu - Centre for Security, Information and Advanced Technologies (CEBIA-Tech), Faculty of Applied Informatics, Tomas Bata University in Zlín, 760 05, 76001 Zlín, Czech Republic

Mengjie Song - Department of Energy and Power Engineering, School of Mechanical Engineering, Beijing Institute of Technology, Beijing 100081, China

Qingbin Gao - School of Mechanical Engineering and Automation, Harbin Institute of Technology Schenzhen, Guangdong 518055, China

Shanti S. Kandala - Department of Chemical and Petroleum Engineering, University of Calgary, Calgary AB T2N 4H9, Canada

Ondřej Kadľćík - TEAZ s.r.o., Otrokovice 765 02, Czech Republic

Complete contact information is available at:

https://pubs.acs.org/10.1021/acsomega.1c02239

\section{Notes}

The authors declare no competing financial interest.

\section{ACKNOWLEDGMENTS}

This work was conducted under the Centre for Security, Information and Advanced Technologies (CEBIA - Tech). This assistance is very gratefully acknowledged. The authors would also like to thank the Tomas Bata University in Zlín for providing the financial support in the form of an internal grant Applications of Engineering Informatics - Advanced Automatic Control Methods and Their Applications in Embedded Systems (RVO/CEBIA/2020/001).

\section{NOMENCLATURE}

\begin{tabular}{|c|c|}
\hline $\mathrm{AC}_{i j}(s)$ & entries of $\mathbf{G}_{\mathrm{AC}}(s)$ \\
\hline$C_{i j}(s)$ & entries of $\mathbf{G}_{\mathrm{C}}(s)$ \\
\hline $\mathbb{C}^{-2}$ & open left complex half-plane \\
\hline $\mathbf{d}(t)$ & load disturbance vector \\
\hline $\mathbf{e}(t)$ & control error vector \\
\hline $\mathbf{G}_{\mathrm{AC}}(s)$ & $\begin{array}{l}\text { antidisturbance controller transfer function } \\
\text { matrix }\end{array}$ \\
\hline $\mathbf{G}_{\mathrm{C}}(s)$ & primary controller transfer function matrix \\
\hline $\mathbf{G}_{\mathrm{D}}(s)$ & decoupling subsystem transfer function matrix \\
\hline $\mathbf{G}_{\mathrm{IDC}}(s)$ & inverted decoupler transfer function matrix \\
\hline $\mathbf{G}_{\mathrm{S}}(s)$ & $\begin{array}{l}\text { controlled system }(\mathbf{u}(t) \text { to } \mathbf{y}(t)) \text { transfer } \\
\text { function matrix }\end{array}$ \\
\hline$\overline{\mathbf{G}}_{\mathrm{S}}(s)$ & $\begin{array}{l}\text { desired open-loop controlled transfer function } \\
\text { matrix }\end{array}$ \\
\hline $\mathbf{G}_{\mathrm{SD}}(s)$ & $\begin{array}{l}\text { disturbance-to-output }(\mathbf{d}(t) \text { to } \mathbf{y}(t)) \text { transfer } \\
\text { function matrix }\end{array}$ \\
\hline I & unit matrix of the particular dimension \\
\hline $\operatorname{IDC}_{i j}(s)$ & entries of $\mathbf{G}_{\mathrm{IDC}}(s)$ \\
\hline$K, K_{\mathrm{p}}$ & $\begin{array}{l}\text { controlled system (static) and controller gains, } \\
\text { respectively }\end{array}$ \\
\hline $\mathbf{r}(t)$ & reference signal vector \\
\hline $\mathbb{R}^{n}$ & n-dimensional Euclidean space \\
\hline$s$ & Laplace transform variable \\
\hline$S_{i j}(s)$ & entries of $\mathbf{G}_{\mathrm{S}}(s)$ \\
\hline $\bar{S}_{i j}(s)$ & entries of $\overline{\mathbf{G}}_{S}(s)$ \\
\hline $\mathrm{Sc}_{i j}(s)$ & cofactor of $S_{i j}(s)$ \\
\hline $\mathrm{SD}_{i j}(s)$ & entries of $\mathbf{G}_{\mathrm{SD}}(s)$ \\
\hline$T, T_{\mathrm{D}}$, and $T_{\mathrm{I}}$ & $\begin{array}{l}\text { controlled system, derivative, and integral time } \\
\text { constants, respectively }\end{array}$ \\
\hline$T_{\mathrm{ar}}$ & average residence time \\
\hline$T_{\mathrm{s}}$ & sampling period \\
\hline$T_{\mathrm{W}}$ & desired control system time constant \\
\hline $\mathbf{u}(t)$ & manipulated input vector \\
\hline $\mathbf{y}(t)$ & controlled output vector \\
\hline$z$ & transform variable \\
\hline
\end{tabular}

\section{GREEK SYMBOLS}

$\bar{\Delta}(s)$ desired control feedback characteristic polynomial

$\tau \quad$ controlled plant time delay

$\theta$ normalized time delay

\section{ABBREVIATIONS}

ARX autoregressive with exogenous

AI, AO analog inputs, analog outputs

DI, DO digital inputs, digital outputs

HMI human-machine interface

IAE integral absolute error

ISE integral square error

ITAE integral time absolute error

LMS least mean square

NRGA normalized relative gain array 
PC personal computer

PCI peripheral component interconnect

PLC programmable logic controller

PID proportional-integral-derivative

PWM pulse width modulation

RGA relative gain array

SCADA supervisory control and data acquisition

SISO single-input single-output

TITO two-input two-output

MIMO multi-input multi-output

TTL transistor-transistor logic

\section{REFERENCES}

(1) Bergman, T. L.; Lavine, A. S.; Incropera, F. P.; Dewitt, D. P. Fundamentals of Heat and Mass Transfer, 7th ed.; John Wiley \& Sons: Hoboken, NJ, 2011.

(2) Pekař, L. Introduction to heat exchangers. In Advanced Analytic and Control Techniques for Thermal Systems with Heat Exchangers, 1st ed.; Pekař, L., Ed.; Academic Press, Elsevier: Cambridge, MA, 2020; pp 3-20.

(3) Corwin, J. F. A hot-air apparatus dryer for general laboratory use. Science 1947, 106, 353.

(4) Getahun, S.; Ambaw, A.; Delele, M.; Meyer, C. J.; Opara, U. L. Analysis of airflow and heat transfer inside fruit packed refrigerated shipping container: Part II - Evaluation of apple packaging design and vertical flow resistance. J. Food Eng. 2017, 203, 83-94.

(5) Rao, D. V. S.; Shivashankara, K. S. Individual shrink wrapping extends the storage life and maintains the antioxidants of mango (cvs. 'Alphonso' and 'Banganapalli') stored at $8{ }^{\circ} \mathrm{C}$. J. Food Sci. Technol. 2015, 52, 4351-4359.

(6) Hot Air Tunnel. https://www.epackagingsrl.com/products/hotair-cutting-tunnel (accessed Dec 15, 2020).

(7) Hot Air Tunnel. http://www.nortan.it/sleeve/hot-air-tunnel/ ?lang=en (accessed Dec 15, 2020).

(8) Honzíček, J. Curing of air-drying paints: A critical review. Ind. Eng. Chem. Res. 2019, 58, 12485-12505.

(9) Suriyachai, P.; Thavarungkul, N.; Saeoui, P. Effects of azodicarbonamide and vulcanization methods on acrylonitrilebutadiene rubber/polyvinyl chloride foam properties. Rubber Chem. Technol. 2013, 86, 86-95.

(10) Visakh, P. M.; Thomas, S.; Chandra, A. K.; Mathew, A. P. Advances in Elastomers I: Blends and Interpenetrating Networks; Springer: Berlin, Heidelberg, Germany, 2013.

(11) Jildeh, Z. B.; Wagner, P. H.; Schöning, M. J. Sterilization of objects, products, and packaging surfaces and their characterization in different fields of industry: The status in 2020. Phys. Status Solidi A 2021, No. 2000732.

(12) Morales-Delgado, D. Y.; Téllez-Medina, D. I.; Rivero-Ramírez, N. L.; Arellano-Cárdenas, S.; López-Cortez, S.; Hérnandez-Sánchez, H.; Gutiérrez-López, G.; Cornejo-Mazón, M. Effect of convective drying on total anthocyanin content, antioxidant activity and cell morphometric parameters of strawberry parenchymal tissue. Rev. Mex. Ing. Quim. 2014, 13, 179-187.

(13) Doymaz, I. Convective drying kinetics of strawberry. Chem. Eng. Process. 2008, 47, 914-919.

(14) El-Mesery, H. S.; Mwithiga, G. Performance of a convective, infrared and combined infrared- convective heated conveyor-belt dryer. J. Food Sci. Technol. 2015, 52, 2721-2730.

(15) Mihindukulasuriya, S. D.; Jayasuriya, H. P. W. Drying of chilli in a combined infrared and hot air rotary dryer. J. Food Sci. Technol. 2015, 52, 4895-4904.

(16) Giri, S. K.; Prasad, S. Drying kinetics and rehydration characteristics of microwave-vacuum and convective hot air dried button mushrooms. J. Food Eng. 2007, 78, 512-521.

(17) Loha, C.; Das, R.; Choudhury, B.; Chatterjee, P. K. Evaluation of air drying characteristics of sliced ginger (Zingiber officinale) in a forced convective cabinet dryer and thermal conductivity measurement. J. Food Process. Technol. 2012, 03, 1-5.
(18) Stoforos, G. N.; Rezaei, F.; Simunovic, J.; Sandeep, K. P. Enhancement of continuous flow cooling using hydrophobic surface treatment. J. Food Eng. 2021, 300, No. 110524.

(19) Silva Júnior, M. A. V.; Rabi, J. A.; Ribeiro, R.; Dacanal, G. C. Modeling of convective drying of cornstarch-alginate gel slabs. J. Food Eng. 2019, 250, 9-17.

(20) Mujumdar, A. S. Drying principles and practice. In Albright's Chemical Engineering Handbook; Albright, L. F., Ed.; CRC: Boca Raton, FL, 2008; pp 1667-1716.

(21) Rashid, M. T.; Ma, H.; Jatoi, M. A.; Hashim, M. M.; Wali, A.; Safdar, B. Influence of ultrasonic pretreatment with hot air drying on nutritional quality and structural related changes in dried sweet potatoes. Int. J. Food Eng. 2019, 15, No. 20180409.

(22) Hajjaji, S. E. Biorefining of waste coffee grounds: Turning an environmental problem into an opportunity. In IOP Conference Series: Earth and Environmental Science,505, 2020 6th International Conference on Environment and Renewable Energy, Hanoi, Vietnam, 2020; No. 012026.

(23) Ghavidel, R. A.; Davoodi, M. G. Studies on physiochemical properties of tomato powder as affected by different dehydration methods and pretreatments. World. Acad. Sci. Eng. Technol. 2010, 69, 596-605.

(24) Yilmaz, M. S.; Şakiyan, Ö.; Barutcu Mazi, I.; Mazi, B. G. Phenolic content and some physical properties of dried broccoli as affected by drying method. Food Sci. Technol. Int. (London, U. K.) 2019, 25, 76-88.

(25) Precoppe, M.; Chapuis, A.; Müller, J.; Abass, A. Tunnel dryer and pneumatic dryer performance evaluation to improve small-scale cassava processing in Tanzania. J. Food Process Eng. 2017, 40, No. e12274.

(26) Verma, M.; Singh, J.; Kaur, D.; Mishra, V.; Rai, G. K. Effect of various dehydration methods and storage on physicochemical properties of guava powder. J. Food Sci. Technol. 2015, 52, 528-534.

(27) Matušu, R.; Prokop, R.; Dlapa, M. Robust control of temperature in hot-air tunnel. In 2008 16th Mediterranean Conference on Control and Automation, Ajaccio, France, 2008; 576-581.

(28) Matušů, R.; Prokop, R. Control of air-flow speed in laboratory model of hot-air tunnel. Procedia Eng. 2015, 100, 345-349.

(29) Pivoňka, P.; Nepevný, P. Hot-air tunnel control using multidimensional predictive controller based on neural network model. In Annals of DAAAM and Proceedings of the International DAAAM Symposium, Vienna, Austria, 2006; 267-268.

(30) Bogdan, S.; Birgmajer, B.; Kovačíc, Z. Model predictive and fuzzy control of a road tunnel ventilation system. Transp. Res. Part $C$ Emerg. Technol. 2008, 16, 574-592.

(31) Oka, Y.; Atkinson, G. T. Control of smoke flow in tunnel fires. Fire Saf. J. 1995, 25, 305-322.

(32) Feng, S.; Li, Y.; Hou, Y.; Li, J.; Huang, Y. Study on the critical velocity for smoke control in a subway tunnel cross-passage. Tunn. Undergr. Space Technol. 2020, 97, No. 103234.

(33) Chaabat, F.; Salizzoni, P.; Creyssels, M.; Mos, A.; Wingrave, J.; Correia, H.; Marro, M. Smoke control in tunnel with a transverse ventilation system: An experimental study. Build. Environ. 2020, 167, No. 106480.

(34) Kondratov, A. P.; Volinsky, A. A.; Chen, J. Macro-mechanism of polyvinyl chloride shrink sleeves embossed marking. J. Appl. Polym. Sci. 2016, 133, No. 43691.

(35) Choab, N.; Allouhi, A.; El Maakoul, A.; Kousksou, T.; Saadeddine, S.; Jamil, A. Review on greenhouse microclimate and application: Design parameters, thermal modeling and simulation, climate controlling technologies. Sol. Energy 2019, 191, 109-137.

(36) Chen, L.; Du, S.; He, Y.; Liang, M.; Xu, D. Robust model predictive control for greenhouse temperature based on particle swarm optimization. Inf. Process. Agric. 2018, 5, 329-338.

(37) Jung, D.-H.; Kim, H.-J.; Kim, J. Y.; Lee, T. S.; Park, S. H. Model predictive control via output feedback neural network for improved multi-window greenhouse ventilation control. Sensors 2020, 20, No. 1756. 
(38) Nachidi, M.; Rodríguez, F.; Tadeo, F.; Guzman, J. L. TakagiSugeno control of nocturnal temperature in greenhouses using air heating. ISA Trans. 2011, 50, 315-320.

(39) Villarreal-Guerrero, F.; Kacira, M.; Fitz-Rodríguez, E.; Linker, R.; Kubota, C.; Giacomelli, G. A.; Arbel, A. Simulated performance of a greenhouse cooling control strategy with natural ventilation and fog cooling. Biosyst. Eng. 2012, 111, 217-228.

(40) Del Sagrado, J.; Sánchez, J. A.; Rodríguez, F.; Berenguel, M. Bayesian networks for greenhouse temperature control. J. Appl. Logic 2016, 17, 25-35.

(41) Liu, L.; Tian, S.; Xue, D.; Zhang, T.; Chen, Y. Q.; Zhang, S. A review of industrial MIMO decoupling control. Int. J. Control Autom. Syst. 2019, 17, 1246-1254.

(42) Bakule, L. Decentralized control: An overview. Annu. Rev. Control 2008, 32, 87-98.

(43) Hu, W.; Cai, W. J.; Xiao, G. Decentralized control system design for MIMO processes with integrators/differentiators. Ind. Eng. Chem. Res. 2010, 49, 12521-12528.

(44) Kadhim, A. M. H. Selection of Decentralized Control Configuration for Uncertain Systems. Ph.D. Thesis, Luleå University of Technology, Department of Computer Science, Electrical and Space Engineering, Luleå, Sweden, 2018.

(45) Mahapatro, S. R.; Subudhi, B.; Ghosh, S. Design and experimental realization of a robust decentralized PI controller for a coupled tank system. ISA Trans. 2019, 89, 158-168.

(46) Garrido, J.; Vázquez, F.; Morilla, F. Centralized inverted decoupling control. Ind. Eng. Chem. Res. 2013, 52, 7854-7866.

(47) Garrido, J.; Vázquez, F.; Morilla, F. Multivariable PID control by decoupling. Int. J. Syst. Sci. 2016, 47, 1054-1072.

(48) Noeding, M.; Martensen, J.; Lemke, N.; Tegethoff, W.; Koehler, J. Selection of decoupling control methods suited for automated design for uncertain TITO processes. In 2018 IEEE 14th International Conference on Control and Automation (ICCA), Anchorage, AK, USA, 2018; 498-505.

(49) Wang, Q. G. Decoupling Control; Springer: Berlin, Heidelberg, Germany, 2003.

(50) Gagnon, E.; Pomerleau, A.; Desbiens, A. Simplified, ideal or inverted decoupling? ISA Trans. 1998, 37, 265-276.

(51) Jevtović, B. T.; Mataušek, M. R. PID controller design of TITO system based on ideal decoupler. J. Process Control 2010, 20, 869-876.

(52) Hamdy, D. M.; Ramadan, A.; Abozalam, B. Comparative study of different decoupling schemes for TITO binary distillation column via PI controller. IEEE/CAA J. Autom. Sin. 2018, 5, 869-877.

(53) Lee, J.; Hyun Kim, D.; Edgar, T. F. Static decouplers for control of multivariable processes. AIChE J. 2005, 51, 2712-2720.

(54) Rajapandiyan, C.; Chidambaram, M. Controller design for MIMO processes based on simple decoupled equivalent transfer functions and simplified decoupler. Ind. Eng. Chem. Res. 2012, 51, 12398-12410.

(55) Wade, H. L. Inverted decoupling: A neglected technique. ISA Trans. 1997, 36, 3-10.

(56) Giraldo, S. A. C.; Flesch, R. C. C.; Normey-Rico, J. E.; Sejas, M. Z. P. A method for designing decoupled filtered Smith predictors for square MIMO systems with multiple time delays. IEEE Trans. Ind. Appl. 2018, 54, 6439-6449.

(57) Li, M.; Zhou, P. Analytical design based hierarchical control for non-square MIMO wood-chip refining process. ISA Trans. 2019, 90, 52-63.

(58) Chuong, V. L.; Vu, T. N. L.; Truong, N. T. N.; Jung, J. H. An analytical design of simplified decoupling Smith predictors for multivariable processes. Appl. Sci. 2019, 9, 2487.

(59) Jain, A.; Babu, B. V. Sensitivity of relative gain array for processes with uncertain gains and residence times. IFAC-PapersOnLine 2016, 49, 486-491.

(60) Bristol, E. On a new measure of interaction for multivariable process control. IEEE Trans. Autom. Control 1966, 11, 133-134.
(61) McAvoy, T. J.; Arkun, Y.; Chen, R.; Robinson, D.; Schnelle, P. D. A new approach to defining a dynamic relative gain. Control Eng. Pract. 2003, 11, 907-914.

(62) Balestrino, A.; Crisostomi, E.; Landi, A.; Menicagli, A. ARGA loop pairing criteria for multivariable systems. In Proceedings of the 47th IEEE Conference on Decision and Control (CDC '08), Cancun, Mexico, 2008; 5668-5673.

(63) He, M. J.; Cai, W. J.; Ni, W.; Xie, L. H. RNGA based control system configuration for multivariable processes. J. Process Control 2009, 19, 1036-1042.

(64) Liao, Q.; Sun, D. Interaction measures for control configuration selection based on interval type-2 Takagi-Sugeno fuzzy model. IEEE Trans. Fuzzy Syst. 2018, 26, 2510-2523.

(65) Navrátil, P.; Pekař, L.; Matušu, R. Control of a multi-variable system using optimal control pairs: A quadruple-tank process. IEEE Access 2020, 8, 2537-2563.

(66) Niederlinski, A. A heuristic approach to the design of linear multivariable interacting control systems. Automatica 1971, 7, 691701.

(67) Gorez, R.; Klán, P. Nonmodel-based explicit design relations for PID controllers. IFAC Proc. Vol. 2000, 33, 133-140.

(68) Vítečková, M.; Víteček, A.; Sladká, K. Controller tuning by desired model method. In 2017 18th International Carpathian Control Conference (ICCC 2017), Sinaia, Romania, 2017; 171-176.

(69) Kučera, V. Diophantine equations in control - A survey. Automatica 1993, 29, 1361-1375.

(70) Grimble, M. J. Robust Industrial Control. Optimal Design Approach for Polynomial Systems; Prentice Hall: London, 1994.

(71) System Identification Toolbox. https://www.mathworks.com/ help/ident/index.html (accessed Feb 01, 2021).

(72) Bobál, V.; Böhm, J.; Fesl, J.; Macháček, J. Digital Self-tuning Controllers: Algorithms, Implementation and Applications; Springer: London, 2005.

(73) Chapellat, H.; Dahleh, M.; Bhattacharyya, S. P. Robust stability under structured and unstructured perturbations. IEEE Trans. Autom. Control 1990, 35, 1100-1108.

(74) Skogestad, S.; Postlethwaite, I. Multivariable Feedback Control: Analysis and Design; Wiley: New York, 2007; Vol. 2.

(75) PCI-1711-100 kS/s, 12-bit, 16-ch Universal Multifunction PCI Card - Advantech. https://www.advantech.com/products/1-

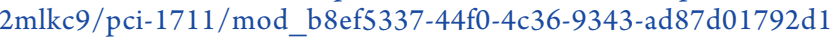
(accessed Feb 02, 2021).

(76) Control Web Software System. https://www.mii.cz/cat?id= 146\&lang $=409$ (accessed Feb 02, 2021).

(77) Pekař, L.; Prokop, R. Algebraic robust control of a closed circuit heating-cooling system with a heat exchanger and internal loop delays. Appl. Therm. Eng. 2017, 113, 1464-1474. 